\title{
Gestão do desempenho em cadeias de suprimentos usando lógica fuzzy
}

\author{
A fuzzy logic approach to supply \\ chain performance management
}

\author{
Gilberto Miller Devós Ganga' \\ Luiz Cesar Ribeiro Carpinetti ${ }^{2}$ \\ Paulo Rogério Politano ${ }^{1}$
}

\begin{abstract}
Resumo: Este trabalho apresenta e discute uma proposta baseada na teoria dos conjuntos fuzzy para predizer o desempenho de uma cadeia de suprimentos modelada de acordo com os relacionamentos causais entre medidas de desempenho propostas pelo SCOR (versão 8.0). O uso de sistemas de medição de desempenho para gerenciar o desempenho de cadeias de suprimentos apresenta algumas limitações tais como a dificuldade de interpretação de resultados de natureza qualitativa, assim como a complexidade de um sistema tradicional de medição de desempenho lidar adequadamente com os relacionamentos causais entre métricas de desempenho de diferentes processos de negócios ao longo da cadeia de suprimentos. Por outro lado, a Lógica Fuzzy, uma técnica apropriada para lidar com situações de incerteza e subjetividade, configura-se como uma alternativa interessante. Utilizando uma abordagem de pesquisa quantitativa descritiva, assumiu-se a hipótese de que um modelo de predição quantitativo poderia ser construído para explicar (no mínimo em parte) o comportamento de processos operacionais. Os resultados do modelo mostraram-se bastante consistentes à metodologia SCOR mark, proposta pelo Supply Chain Council. Análises estatísticas dos resultados, baseados no Método de Superfície de Resposta, também confirmaram a relevância dos relacionamentos causais incorporados no modelo. Em geral, os resultados reforçam que a proposição da adoção de um modelo de predição baseado em lógica fuzzy e nas métricas do SCOR parece ser uma abordagem possível para auxiliar os gerentes no processo de tomada de decisão do gerenciamento do desempenho em cadeias de suprimentos.

Palavras-chave: Gestão da cadeia de suprimentos. Modelo SCOR. Medidas de desempenho. Lógica fuzzy. Modelo de predição.
\end{abstract}

\begin{abstract}
Supply chain measurement is a complex activity due to the broad taxonomy of variables (quantitative or qualitative; financial or non-financial, and others). This scenario results in a vague and subjective process to evaluate performance. Accordingly, the fuzzy logic seems to be a viable approach to measure Supply Chain Performance. This work proposes the creation of a simulation environment, developed on FuzzyTECH platform. It is based on a hierarchical model of SCOR metrics, which aims the assessment of the supply chain performance. The goal of such model is to evaluate systemically the supply chain performance establishing the cause-effect relation can be between performance attributes and levels 1 and 2 of the SCOR metrics. The findings showed great consistency when compared to the SCORmark approach suggested by the Supply Chain Council for a benchmarking process. The model is expected to be a useful tool in the supply chain performance diagnosis and improvement process contributing to strategic supply chain management decision-making processes
\end{abstract}

Keywords: Supply chain metrics. Model SCOR. Performance evaluation system. Fuzzy logic. SCOR simulation.

\section{Introdução}

A gestão do desempenho de cadeias de suprimentos tem-se tornado uma atividade de reconhecida importância, principalmente pela complexa natureza dos processos de negócios, geralmente envolvendo múltiplos critérios de decisão (CAI; LIU; LIU,

2009; YEH; CHENG; CHI, 2007). Pesquisas sobre a aplicação de sistemas de medição de desempenho para gestão de desempenho de cadeias de suprimentos são citadas em inúmeros trabalhos. É o caso da aplicação do Balanced Scorecard - BSC (BREWER; SPEH,

\footnotetext{
${ }^{1}$ Departamento de Engenharia de Produção, Universidade Federal de São Carlos - UFSCar, Rod. Washington Luís, Km 235, CEP 13565-905, São Carlos, SP, Brasil, e-mail: ganga@dep.ufscar.br; paulo@dc.ufscar.br

${ }^{2}$ Departamento de Engenharia de Produção, Escola de Engenharia de São Carlos - EESC, Universidade de São Paulo - USP, Av. Trabalhador são-carlense, 400, CEP 13566-590, São Carlos, SP, Brasil, e-mail: carpinet@ @sc.usp.br
}

Recebido em 20/10/2019 - Aceito em 10/5/2011

Suporte financeiro: Nenhum. 
2000; KLEIJNEN; SMITS, 2003; PARK; LEE; YOO, 2005). Alguns autores ainda propõem métricas voltadas à cadeia de suprimentos (BEAMON, 1999; BEAMON; CHEN, 2001; GUNASEKARAN et al., 2001, 2004; SHEPHERD; GÜNTER, 2006). Estas abordagens identificam relações causais entre as métricas de desempenho, porém, não as quantificam.

Outras abordagens para avaliação de desempenho incluem modelos multicritério, como o Analytic Hierarchy Process - AHP (CHAN, 2003; AGARWAL; SHANKAR;TIWARI, 2006), ou o uso combinado do BSC-AHP (SHARMA; BHAGWAT, 2007; THAKKAR et al., 2007; VARMA; WADHWA; DESHMUKH, 2008) e programação linear (BELTON; STEWART, 2002; SANTOS; BELTON; HOWICK, 2002, 2008; GAUDENZI; BORGHESI, 2006; SEYDEL, 2006; AISSAOUI; HAOUARI; HASSINI, 2007; WEBER et al., 2008; ORDOOBADI, 2009). A programação linear apresenta algumas limitações, tais como a necessidade de se trabalhar com dados históricos para identificação das funções de distribuição de probabilidades das variáveis envolvidas e a dificuldade em interpretar resultados de natureza qualitativa, no sentido de uma avaliação sistêmica da cadeia de suprimentos. O Analytic Hierarchy Process (AHP), por sua vez, pode avaliar métricas de natureza qualitativa. No entanto, a quantificação dos relacionamentos causais por meio da escala de comparação pareada (escala saaty) está sujeita a erros de julgamento humano (JAIN;TIWARI; CHAN, 2004). Por outro lado, a lógica fuzzy é apropriada para lidar com situações de incerteza e subjetividade (ZADEH, 1978), típicas de medidas de natureza qualitativa e comparação pareada, como o AHP.

A lógica fuzzy tem sido cada vez mais empregada no processo de apoio à tomada de decisões (MACHACHA; BHATTACHARYA, 2000; LIN; CHEN, 2004; MON; CHENG; LIN, 1994; LIN; CHIU; CHU, 2006). A utilização de conjuntos fuzzy com fins de avaliação de desempenho em cadeias de suprimentos é mencionada em alguns trabalhos (CHAN et al., 2003; CHAN; QI, 2003; GASPARETTO, 2003; JESUS, 2003; PIRES; ARAVECHIA, 2001; PIRES, 2004; SELLITTO; MENDES, 2006; YEH; CHENG; CHI, 2007; THEERANUPHATTANA; TANG, 2008). Unahabhokha, Platts e Tan (2007) destacam ainda a relevância do desenvolvimento de um sistema de medição baseado em lógica fuzzy para predizer o desempenho organizacional.

Portanto, este trabalho objetiva apresentar e discutir uma proposta baseada na teoria dos conjuntos fuzzy para predizer o desempenho de uma cadeia de suprimentos modelada de acordo com os relacionamentos causais entre medidas de desempenho propostas pelo Supply Chain Operations Reference (Modelo SCOR, versão 8.0). O modelo SCOR foi escolhido por incorporar alguns processos de negócios-chave ao longo da cadeia de suprimentos, reforçando a necessidade de se desenvolver uma avaliação de desempenho sistêmico (HOLMBERG, 2000). Para tanto, a lógica fuzzy constitui uma boa alternativa para quantificar os relacionamentos de causa e efeito das métricas de desempenho em todos os níveis e subníveis dos processos de negócios que constituem uma cadeia de suprimentos.

Theeranuphattana e Tang (2008) propuseram um modelo conceitual, que também utilizou a lógica fuzzy e o modelo SCOR para a gestão de desempenho em cadeia de suprimentos. No entanto, o modelo restringiu-se apenas a métricas de nível 1 do SCOR. $O$ modelo de predição apresentado neste artigo inclui as métricas de nível 2 do SCOR, o que ressalta a contribuição original da proposta.

Este trabalho está organizado da seguinte maneira: A seção 2 revisa brevemente conceitos sobre medição de desempenho em cadeias de suprimentos e o modelo SCOR, o modelo de referência usado neste artigo. A seção 3 visa a esclarecer alguns conceitos fundamentais sobre os sistemas fuzzy como uma abordagem útil ao processo de predição de desempenho em cadeias de suprimentos. A seção 4 descreve o modelo de predição proposto, enquanto a seção 5 apresenta os resultados do modelo de predição. A seção 6 apresenta a análise dos resultados gerados pelo modelo, baseada na metodologia de superfície de resposta. Finalmente, a seção 7 tece as considerações finais sobre a pesquisa realizada.

\section{Medição de desempenho em cadeias de suprimentos}

A medição de desempenho no contexto de cadeias de suprimentos tem se tornado extremamente importante. A razão para isso é evidente: as organizações estão procurando formas para melhorar seu desempenho operacional por meio de melhor integração das operações na cadeia de valor. A habilidade para medir e avaliar o desempenho das operações pode ser vista como uma necessidade para as empresas, que gastam enormes recursos ao longo dos anos para desenvolver sistemas de medição de desempenho com tal fim (KUENG, 2000).

O principal benefício de um sistema de gestão do desempenho para cadeias de suprimentos é fornecer um quadro compreensível e atual de informações sobre o desempenho de um negócio. Outra contribuição é possibilitar um diagnóstico das fraquezas do negócio e decidir quando e onde ações corretivas se tornam necessárias, a fim de avaliar o impacto dessas ações sobre o desempenho do todo (KUENG; WETTSTEIN; LIST, 2001).

A natureza complexa dos processos de negócios que compõem uma cadeia de suprimentos resulta numa atividade de avaliação de desempenho extremamente 
complexa, constituindo-se uma problemática que geralmente envolve múltiplos critérios. A avaliação e o emprego de múltiplas medidas de desempenho têm sido encorajados por inúmeros trabalhos sobre medição de desempenho (van HOEK, 1998; BEAMON, 1999; HOLMBERG, 2000; LAPIDE, 2000; DE TONI; TONCHIA, 2001; GUNASEKARAN, et al., 2001; LAMBERT; POHLEN, 2001; STEPHENS, 2001; BULLINGER; KUHNER, 2002; HIEBER; VAN HOFF, 2002; CHAN, 2003; CHAN; QI, 2003; KLEIJNEN; SMITS, 2003; GUNASEKARAN; PATEL; MCGAUGHEY, 2004; HUANG; SHEORAN; WANG, 2004; LOCKAMY; MCCORMACK, 2004; MORGAN, 2004; SCHONSLEBEN, 2004; HERVANI; HELMS; SARKIS, 2005; LI et al., 2005; ZHU; SARKIS; GENG, 2005; ANGERHOFER; ANGELIDES, 2006; BLINGE; SWENSSON, 2006; SHEPHERD; GÜNTER, 2006; BOLSTORFF, 2007; BRINDLEY; RITCHIE, 2007; ZHU; SARKIS; LAI, 2008; CUTHBERTSON; PIOTROWICZ, 2008). Todavia, quando um número de medidas é avaliado simultaneamente, observa-se que os gerentes não são capazes de distinguir quando um processo particular é influenciado por outro propriamente dito (GOLEÇ; TASKIN, 2007). É necessário, portanto, o desenvolvimento de abordagens de gestão do desempenho que possam conciliar critérios de desempenho com diversos aspectos necessários aos tomadores de decisão dessas organizações ou rede de empresas (YEH; CHENG; CHI, 2007).

No Brasil, alguns trabalhos sobre medição de desempenho em cadeias de suprimentos podem ser citados. Gasparetto (2003) propõe um sistema de medição de desempenho para a cadeia de suprimentos. Jesus (2004) analisa a medição de desempenho na cadeia de suprimentos de uma montadora de automóveis. Ambos os trabalhos, porém, assumem características qualitativas, por não tratarem especificamente da quantificação das relações causais das medidas de desempenho envolvidas no processo de medição da Supply Chain (SC). Pires e Aravechia (2001) propõem uma lógica baseada em gráficos-radar para a avaliação do desempenho de SCs de unidades de negócio isoladas. No entanto, tal trabalho não promove uma estrutura sistêmica de avaliação do desempenho da cadeia de suprimentos (HOLMBERG, 2000) para analisar quantitativamente os relacionamentos de causa e efeito entre medidas de diferentes categorias, níveis e processo, o propósito maior deste trabalho.

Outra pesquisa, de característica quantitativa, realizada no Brasil, foi a proposta por Sellitto e Mendes (2006). O trabalho apresentou um caso de avaliação do desempenho de três cadeias de suprimentos em manufatura. A metodologia proposta partiu do modelo SCOR de gestão de cadeias de suprimento. As ideias do modelo foram estruturadas e associadas a dimensões de desempenho, cujas importâncias relativas foram calculadas por especialistas, com o auxílio do AHP.

As abordagens citadas anteriormente apresentam algumas limitações, como erros de julgamento humano (natureza subjetiva), além da dificuldade de se realizar uma avaliação sistêmica da cadeia de suprimentos, que leve em conta as relações de causa e efeito entre critérios de desempenho e métricas, nos níveis e subníveis dos processos de negócios que compõem uma cadeia de suprimentos.

Duas importantes características para medidas de desempenho podem ser citadas (KUENG, 2000): 1) uma medida de desempenho é basicamente multidimensional, daí a existência de muitos indicadores; 2) não são independentes. Muitos indicadores de desempenho têm uma relação de influência sobre outros. Tais relacionamentos podem ser conflitantes ou complementares. Portanto, a independência seria uma exceção.

Importante se faz ressaltar que, no projeto de um sistema de avaliação de desempenho em cadeias de suprimentos, é a seleção das medidas-chave que avaliam o desempenho da cadeia de suprimentos como um todo. Um conceito interessante, que pode auxiliar este processo, é a hierarquização das métricas de desempenho, proposta por Chang e Morgan (2000). O conceito de hierarquização de métricas de desempenho é, inclusive, utilizado pelo modelo de referência SCOR e será uma das bases do modelo de predição de desempenho de cadeia de suprimentos proposto neste trabalho.

O Supply Chain Operations Reference (SCOR), proposto pelo Supply Chain Council (2009), é um modelo de referência de processos de negócios para a gestão de cadeia de suprimentos. Ele possibilita às empresas determinar e comparar rapidamente $\mathrm{o}$ desempenho da cadeia de suprimentos como um todo. A grande vantagem dessa ferramenta é a criação de uma linguagem comum e padronizada entre os demais agentes que compõem uma determinada cadeia de suprimentos. O modelo fornece um framework que visa a apoiar todos os aspectos envolvidos no desempenho dos agentes que compõem uma cadeia de suprimentos. Por meio de tal arquitetura, é possível mapear as conexões entre os processos interorganizacionais de cada empresa numa supply chain.

O SCOR propõe uma análise da cadeia de suprimentos a partir de três perspectivas: processo, métricas e melhores práticas. Cada perspectiva analisa uma supply chain baseada numa estrutura top-down, por meio da qual é possível desdobrar em níveis e subníveis de análise. A ideia central desta visão hierárquica de processos é relacionar cada operação de processo com as respectivas métricas de desempenho (SUPPLY..., 2009). Tais processos são considerados processos intraorganizacionais críticos dentro de uma cadeia de suprimentos, com 
cinco atributos de desempenho (BOLSTORFF; ROSENBAUM, 2007), conforme segue:

- Confiabilidade: significa o desempenho de uma cadeia de suprimentos na entrega, ou seja, o produto correto, no lugar correto, na quantidade correta, no tempo correto, na integridade correta (qualidade do produto e embalagem), com a documentação correta e o cliente certo;

- Responsividade: está relacionada à velocidade com que uma cadeia de suprimentos fornece os produtos aos clientes;

- Flexibilidade: significa a agilidade de uma cadeia de suprimentos em responder às variações de demanda para ganhar ou manter uma vantagem competitiva;

- Custos: contempla todos os custos relacionados à operação de uma Supply Chain;

- Eficiência na gestão de ativos: está relacionada à eficiência de uma organização no gerenciamento de seus recursos para atender à demanda. Inclui a gestão de todos os recursos: fixos e capital operacional.

Assim como os demais níveis do SCOR são desdobrados em categorias de processos, atividades e tarefas, as medidas de desempenho também o são. Nesse sentido, para cada métrica estabelecida no nível 1, de acordo com os critérios de medição, existe uma hierarquia de métricas inter-relacionadas. $\mathrm{O}$ desdobramento de tais medidas, por meio de relações de causa e efeito, torna possível analisar o desempenho de uma cadeia de suprimentos por várias óticas, desde uma visão mais operacional até uma perspectiva mais estratégica.

A Figura 1 ilustra esse comportamento para a medida de desempenho upside supply chain flexiblity (incremento de flexibilidade na cadeia de suprimentos), relativa ao atributo de desempenho flexibilidade. O Upside Flexibility Supply Chain é uma medida discreta que define a quantidade de tempo, geralmente em dias, que uma cadeia demoraria para responder a uma variação de demanda não planejada em torno de $20 \%$, sem custo ou penalidade de serviço. Ele pode ser desdobrado em três medidas de nível 2 do SCOR: Upside Source Flexibility (incremento de flexibilidade no processo de suprimentos); Upside Make Flexibility (incremento de flexibilidade no processo de fabricação), $e$ Upside Deliver Flexibility (incremento de flexibilidade no processo de distribuição) (SUPPLY ..., 2009). Tais medidas representam a capacidade de resposta (em dias) dos processos suprimentos (source), fabricação (make) e entrega (deliver) em se adequar à variação de demanda, sem custos e penalidades de atendimento do serviço ao cliente. Cada indicador, respectivo ao seu processo, possuirá diferentes características de decisão e restrição para se readequar a tal índice de incremento de demanda.

Por exemplo, a medida Upside Source Flexibility (Figura 1) poderia ser composta por: a) quantidade de tempo necessário para obtenção de capital para ajustar o gap entre a disponibilidade atual de capital e o capital necessário para sustentar o aumento de $20 \%$ nas quantidades pedidas; b) quantidade de tempo necessário para obter e entregar o estoque (matéria-prima ou produtos finais) para processamento do pedido, incluindo estoque de segurança para sustentar os $20 \%$ de aumento nas quantidades a serem supridas, e c) quantidade de tempo necessário para recrutar, contratar e treinar um staff adicional para ajustar o gap entre a atual força de trabalho e o staff necessário para sustentar os $20 \%$ de aumento nas quantidades a serem entregues.

Assim, baseado nesses relacionamentos de causa e efeito, este trabalho propôs a arquitetura de referência do modelo de predição de desempenho da cadeia de suprimentos apresentado na seção 4 .

\section{Funcionamento do modelo fuzzy}

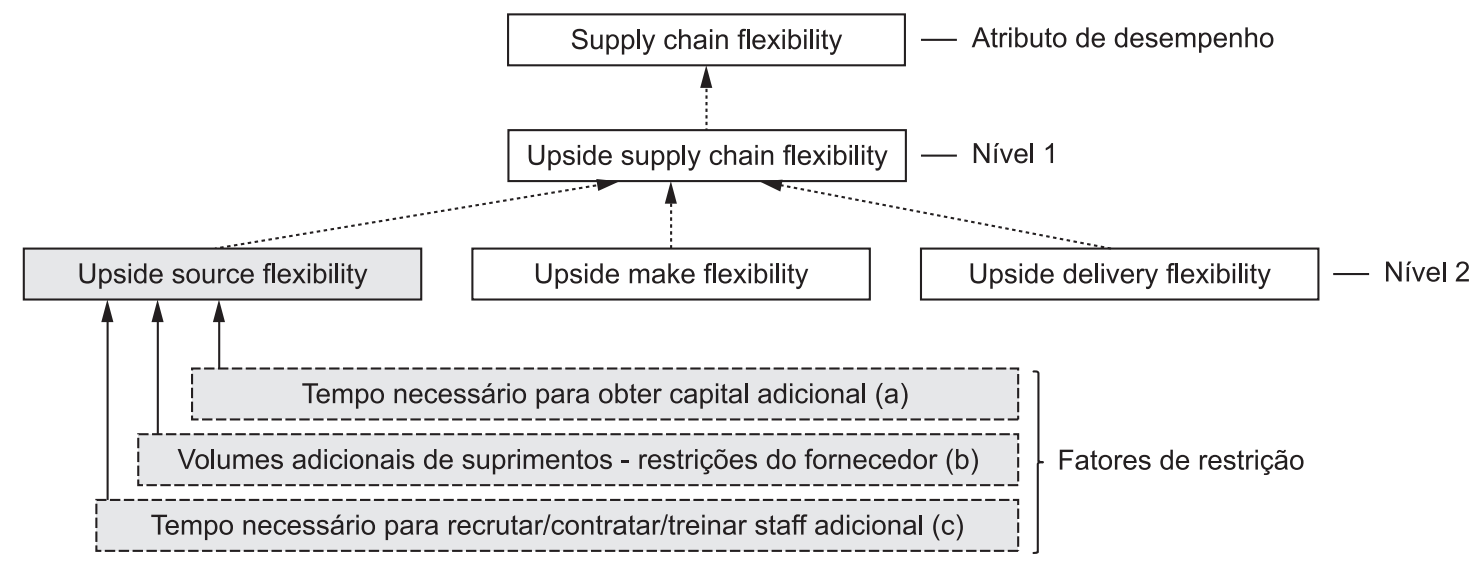

Figura 1. Upside Source Flexibility: estrutura hierárquica da métrica. Fonte: Adaptado de Supply Chain Council (2009). 
Nesta seção, algumas definições básicas sobre lógica fuzzy foram revisadas a partir de Zadeh (1978); Dubois e Prade (1980); Zimmermann (1991); Klir e Yuan (1995); Bojadziev e Bojadziev (1997); Pedrycz e Gomide (1998); Carlsson, Fedrizzi e Fullér (2004); Barros e Bassanezi (2006); Simões e Shaw (2007). Tais definições e notações foram utilizadas em todo o trabalho e são imprescindíveis para a compreensão do modelo proposto.

Definiçãa 1. Um conjunto fuzzy Ã em $X$ é definido por (Equação 1)

$$
\tilde{\mathrm{A}}-\left\{x, \mu_{\mathrm{A}}(x) / x \in \mathrm{X}\right\}
$$

em que $\mu_{\mathrm{A}}(x): X \rightarrow[0,1]$ é chamada de função de pertinência de $\tilde{A}$ e $\mu_{\mathrm{A}}(x)$ é o grau de pertinência de $x \operatorname{em} \tilde{A}$.

Definição 2. Variável linguística: é uma variável cujo valor é expresso qualitativamente por termos linguísticos (que fornecem um conceito à variável) e quantitativamente por uma função de pertinência. O nível de serviço, por exemplo, é uma variável linguística cujos valores podem ser: muito baixo, baixo, médio, alto ou muito alto.

Definição 3. Fuzzificação: Este módulo efetua um mapeamento entre os valores numéricos das variáveis crisp de entrada do sistema para graus de compatibilidade com conceitos linguísticos. A atuação de um especialista na área do fenômeno a ser modelado é de fundamental importância para colaborar na construção das funções de pertinência para a descrição das entradas.

Definição 4. Função de Pertinência (FP): é uma função numérica, gráfica ou tabulada, que atribui valores de pertinência fuzzy para valores discretos de uma variável, em seu universo de discurso, o intervalo numérico de todos os possíveis valores reais que uma variável específica pode assumir.

Definição 4.1 Função de Pertinência Triangular (FPT)

Um número fuzzy Ã é um número triangular fuzzy se sua função de pertinência $\mu_{\mathrm{A}}$ for dada pela Equação 2:

$$
\mu_{\mathrm{A}}(x)=\left\{\begin{array}{c}
0 \text { for } x<a, \\
\frac{x-\mathrm{a}}{\mathrm{b}-\mathrm{a}} \text { for } a \leq x \leq b, \\
\frac{\mathrm{c}-\mathrm{x}}{\mathrm{c}-\mathrm{b}} \text { for } b \leq x \leq c, \\
0 \text { for } x>c,
\end{array}\right.
$$

Em que $a, b$ e $c$ são números reais com $a<b<c$. Fora do intervalo $[a, c]$, o grau de pertinência é nulo, $b$ representa o ponto em que o grau de pertinência é máximo, como pode ser compreendido por meio da Figura 2.

A notação usada para representar as funções de pertinência triangulares, neste trabalho, segue a lógica expressa na Figura 3 a seguir, que exemplifica o uso das funções de pertinência para a medida de desempenho Acurácia na Documentação (Accurate Documentation).

Como pode ser observada, a composição das funções de pertinência dá-se em função dos pontos $\left(x_{i} ; y_{i}\right)$, em que $x_{i}$ denota o universo de discurso da variável e $y_{i}$ o nível de pertinência para uma dada medida. Analisando a Figura 3, percebe-se que os pontos em destaque definem os limites e formas das funções de pertinência triangulares criadas. Os pontos $(84 ; 1)$ e $(91 ; 0)$, definem, por exemplo, a função de pertinência triangular "baixo". Os pontos $(84 ; 0),(91 ; 1)$ e $(98 ; 0)$, definem, por exemplo, a função de pertinência triangular "médio". A função de pertinência "alto" foi definida seguindo o mesmo esquema lógico. Existem diferentes tipos de funções de pertinência, como, por exemplo, a triangular, trapezoidal e gaussiana, mas a função de pertinência triangular é a mais empregada (WU; LEE, 2007; ROSS, 1997; LIU; LIU, 2001), porque é intuitivamente fácil de usar e calcular, por parte dos tomadores de decisão (DUBOIS; PRADE, 1980; LIN; HSU; SHEEN, 2007).

Definição 5. Regras $S e$... Então: A base de regras relaciona logicamente as informações que formam a base de conhecimentos do sistema fuzzy. A partir da definição das funções de pertinência, tanto das variáveis de entrada quanto das variáveis de saída, é possível mapear a base de regras, geralmente a partir da opinião de especialistas. As regras com possíveis saídas fuzzy representadas por $C_{i j}$ são apresentadas simbolicamente por meio de uma matriz $n \times m(n$ linhas e $m$ colunas), chamada quadro de decisão, em que $C_{i j}, i=1, \ldots, \mathrm{n}, \mathrm{j}=1, \ldots, \mathrm{m}$, são renomeados elementos do conjunto $\left\{C_{1}, \ldots, C_{1}\right\}$ como pode ser observado no Tabela 1 .

Dessa maneira, o atual significado de $S e$... então é

$$
\text { Se } x \text { é } A_{\mathrm{i}} \text { e } y \text { é } B_{\mathrm{j}} \text {, então } z \text { é } C_{k}
$$

Definição 6. Máquina de Inferência: Este módulo realiza o processamento fuzzy propriamente dito. Cada proposição fuzzy é traduzida matematicamente por meio das técnicas de "raciocínio aproximado", ou seja, as operações de conjuntos fuzzy. Os operadores matemáticos são selecionados a fim de definir a relação

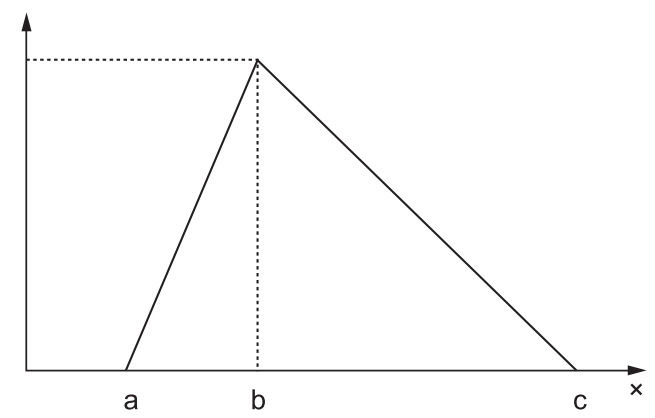

Figura 2. Função de pertinência triangular. 


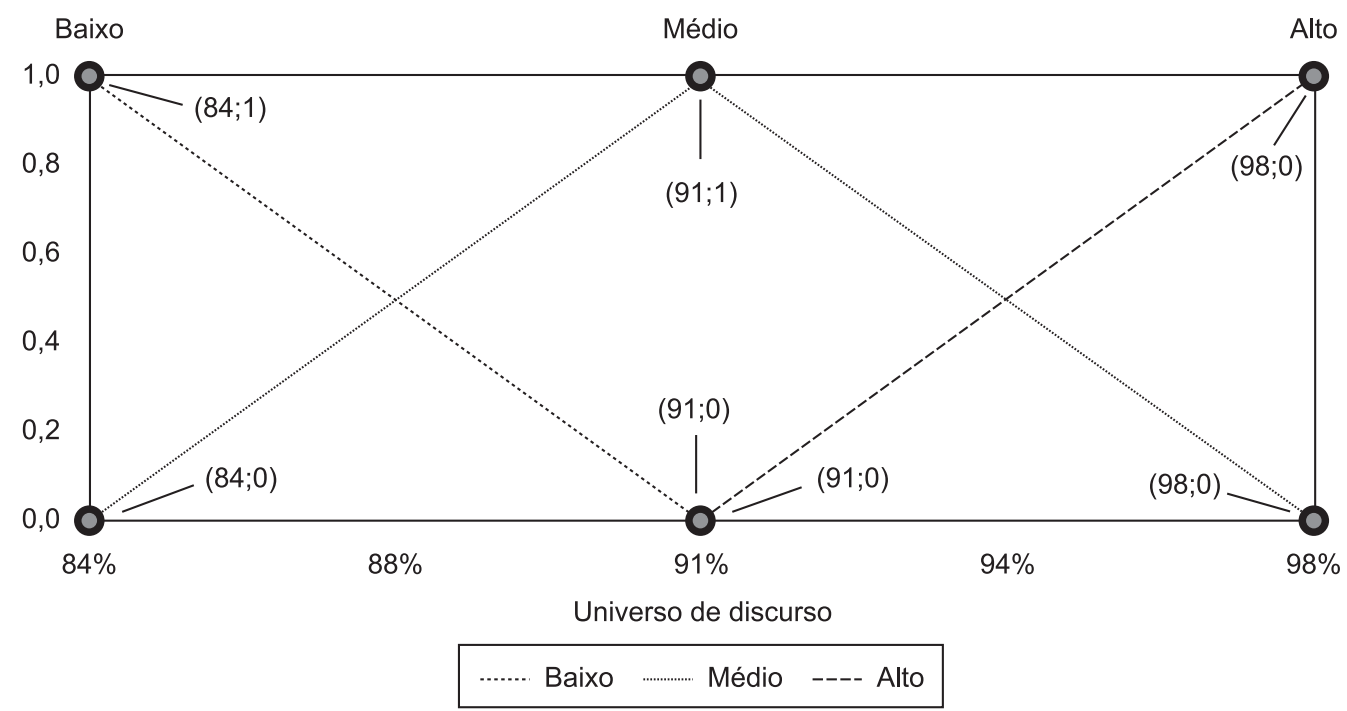

Figura 3. Composição das funções de pertinência para Acurácia na Documentação.

fuzzy que modela a base de regras. A máquina de inferência é fundamental para o sucesso do sistema fuzzy, já que fornece a saída a partir de cada entrada fuzzy e da relação definida pela base de regras. A literatura sobre lógica fuzzy apresenta basicamente dois métodos de inferência fuzzy: o Método de Mamdani e o Método de Kang-Takagi-Sugeno. A diferença básica entre esses dois métodos recai no tipo de variável de resposta (consequente) e no procedimento de fuzzificação. Este trabalho adotou integralmente o método de inferência Mamdani.

Definição 6.1 Método Mamdani

Por meio de uma perspectiva teórica, Mamdani propôs uma relação binária fuzzy $M$ entre $x$ e $\mu$ para modelar matematicamente a base de regras. Tal método é baseado na regra de composição de inferência max - min, seguindo os procedimentos:

- em cada regra $R_{j}$, da base de regras fuzzy, a condicional "se $x$ é $A_{j}$, então $\mu$ é $B_{j}$ " é modelada pela aplicação $\wedge$ (mínimo);

- adota-se a t-norma $\wedge$ (mínimo) para o conectivo lógico "e";

- para o conectivo lógico "ou", adota-se a t-conorma $\vee$ (máximo) que conecta as regras fuzzy da base de regras.

Desse modo, a relação binária $M$ é o subconjunto fuzzy de $M \times U$, cuja função de pertinência é dada por $\varphi M(\mathrm{x}, \mu)=\max _{1 \leq \mathrm{i} \leq \mathrm{r}}\left[\varphi \mathrm{R}_{\mathrm{i}}(\mathrm{x}, \mu)=\max _{1 \leq \mathrm{i} \leq \mathrm{r}}\left[\varphi A_{\mathrm{j}}(\mathrm{x}) \wedge \varphi \mathrm{B}_{\mathrm{j}}(\mu)\right](4)\right.$

Em que $r$ é o número de regras que compõem a base de regras, $\tilde{A}_{j}$ e $\widetilde{B}_{\mathrm{j}}$ são os subconjuntos fuzzy da regra $j$. Cada um dos valores $\varphi A_{\mathrm{j}}(x)$ e $\varphi B_{j}(\mu)$ é interpretado como os graus com que $x$ e $\mu$ estão nos subconjuntos fuzzy, $\tilde{A}_{j}$ e $\widetilde{B}_{\mathrm{j}}$, respectivamente.

Definição 7. Defuzzificação: Este módulo transforma o conceito linguístico, obtido pelo procedimento
Tabela 1. Regras Se ... Então.

\begin{tabular}{ccccccc}
\hline & $B_{1}$ & $\cdots$ & $B_{\mathrm{j}}$ & $B_{\mathrm{j}+1}$ & $\cdots$ & $B_{\mathrm{m}}$ \\
\hline$A_{1}$ & $C_{11}$ & $\cdots$ & $C_{1 \mathrm{j}}$ & $C_{1, \mathrm{j}+1}$ & $\cdots$ & $C_{1, \mathrm{~m}}$ \\
$\vdots$ & $\vdots$ & & $\vdots$ & $\vdots$ & & $\vdots$ \\
$A_{\mathrm{i}}$ & $C_{\mathrm{i} 1}$ & $\cdots$ & $C_{\mathrm{ij}}$ & $\mathrm{C}_{\mathrm{i}, \mathrm{j}+1}$ & $\cdots$ & $C_{\mathrm{i}, \mathrm{m}}$ \\
$\mathrm{A}_{1+1}$ & $C_{\mathrm{i}+1,1}$ & $\cdots$ & $C_{\mathrm{i}+1, \mathrm{j}}$ & $\mathrm{C}_{\mathrm{i}+1, \mathrm{j}+1}$ & $\cdots$ & $C_{\mathrm{i}+1, \mathrm{~m}}$ \\
$\vdots$ & $\vdots$ & & $\vdots$ & $\vdots$ & & $\vdots$ \\
$\mathrm{A}_{\mathrm{n}}$ & $C_{\mathrm{n} 1}$ & $\cdots$ & $C_{\mathrm{nj}}$ & $C_{\mathrm{n}, \mathrm{j}+1}$ & $\cdots$ & $C_{\mathrm{nm}}$ \\
\hline
\end{tabular}

de inferência, em um valor numérico bem definido (variável crisp), o qual é utilizado como a saída efetiva do sistema fuzzy. Os métodos mais conhecidos para a defuzzificação são o método do centro de área (CoA); o método dos centros máximos $(\mathrm{CoM})$; e o método da média dos máximos $(\mathrm{MoM})$, todos utilizados neste trabalho.

\section{$4 \mathrm{O}$ modelo proposto}

A abordagem de pesquisa foi baseada na hipótese de que um modelo de simulação quantitativo poderia ser construído para explicar (no mínimo em parte) o comportamento de processos operacionais numa supply chain e auxiliar na elucidação de problemas de tomada de decisão com os quais os gerentes de supply chain estão envolvidos. Em particular, a pesquisa apresentada neste trabalho caracteriza-se como uma pesquisa quantitativa-empírica-descritiva (BERTRAND; FRANSOO, 2002), a qual se define como uma categoria de pesquisa interessada na criação de um modelo (neste caso, um modelo de simulação) que descreva adequadamente relacionamentos causais que possam existir na realidade, objetivando compreender os processos numa cadeia de suprimentos. 
O modelo proposto neste trabalho usa a lógica fuzzy para estabelecer relações de causalidade entre variáveis de desempenho (métricas) definidas pelo modelo SCOR e, com isso, poder simular o desempenho dos processos de uma cadeia de suprimentos. A Figura 4 ilustra a interação entre o mundo real e a modelagem desse mundo.

O emprego da lógica fuzzy na modelagem do mundo atua como um mecanismo de predição de desempenho das variáveis de resposta (lagging), em função de determinados valores de entrada (leading), que são determinados por meio de estimação ou dados disponíveis. Desta maneira, a lógica fuzzy foi utilizada para modelar o efeito de uma variável de entrada (leading) sobre uma variável resposta (lagging), como pode ser observado na Figura 4.
Por meio do modelo proposto, é possível, por exemplo, predizer o comportamento da medida Upside Source Flexibility (lagging) a partir das medidas (leading) (a) (b) e (c), ilustradas na Figura 1. O desenvolvimento do modelo fuzzy em destaque na Figura 4 pode ser compreendido (passo a passo) por meio do processo descrito na Figura 5.

Passo 1: A definição das métricas de desempenho é uma tarefa crítica no desenvolvimento de um sistema de medição de desempenho. Baseado na premissa da falta de um equacionamento lógico entre as medidas de desempenho antecedentes (leading) e as medidas de desempenho consequentes (lagging), este trabalho recorreu ao modelo SCOR 8.0 a fim de obter uma arquitetura de referência na compreensão dos relacionamentos de causa e efeito entre os atributos

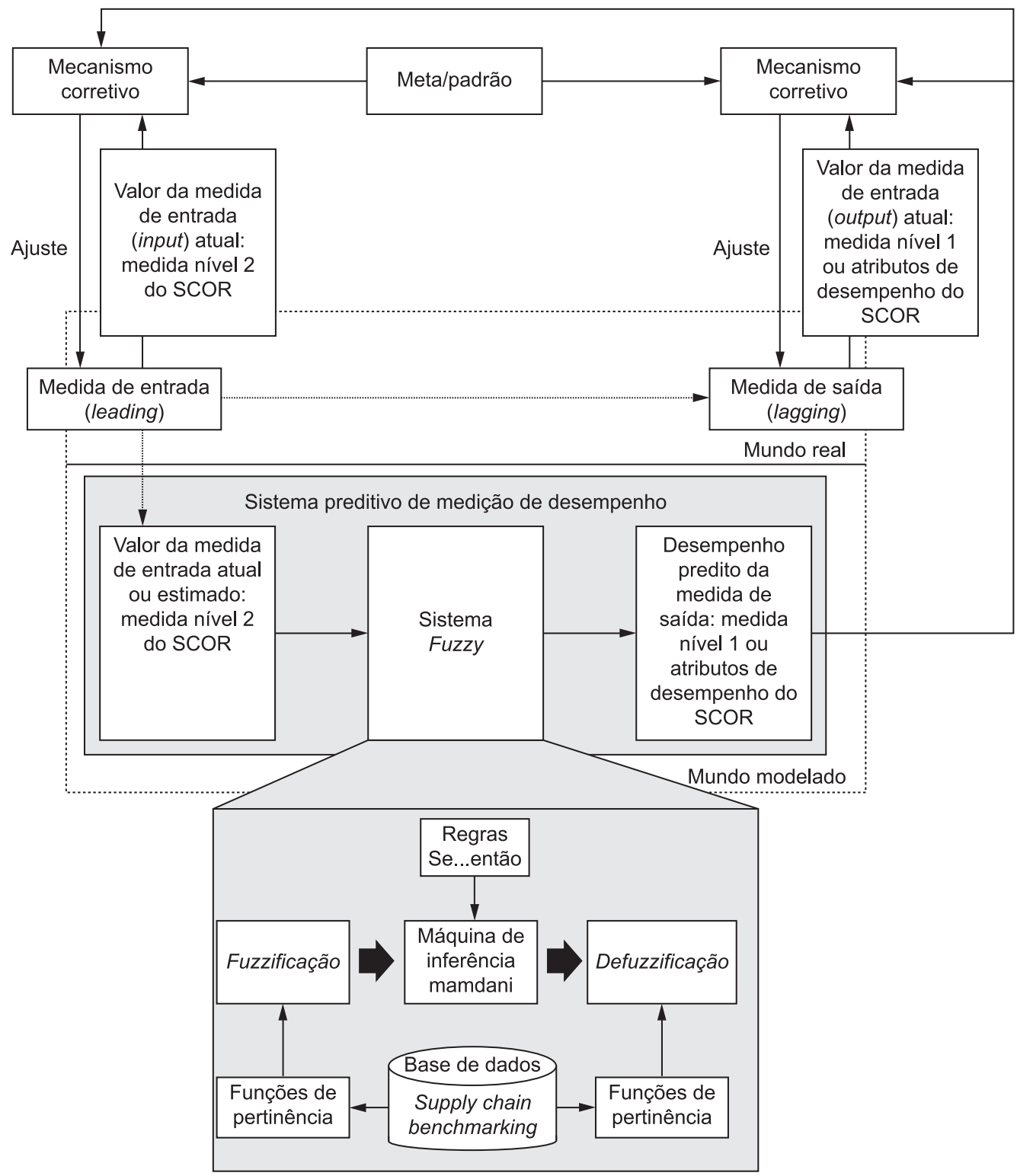

Figura 4. Sistema Preditivo de Medição de Desempenho. Fonte: Proposto pelos autores a partir de Unahabhokha, Platts e Tan (2007). 
de desempenho, medidas de nível 1 e medidas de nível 2, relativas ao desempenho de uma cadeia de suprimentos. O entendimento de tais relações lógicas foi imprescindível na proposição do modelo em questão, como pode ser observado na Figura 6.

O SCOR 8.0, por meio do documento SCORcard, forneceu uma ampla gama de medidas de desempenho (níveis 1,2 e 3) para os atributos de desempenho em cadeia de suprimentos. Seria inviável desenvolver um modelo para a totalidade de métricas sugeridas pelo SCOR 8.0.

Nesse sentido, a escolha das medidas de desempenho, em termos de variedade e quantidade, foi baseada em informações de benchmarking de inúmeras fontes, tais como o Supply Chain Council (portal SCORmark), Productivity and Quality with Performance Measures \& Metrics (APQC) e o Manufacturing Performance Institute (MPI). Cada uma das métricas incluídas no modelo (Figura 6) foi modelada por meio de uma função fuzzy, como detalhado a seguir.

\subsection{Desempenho da cadeia de suprimentos em custos}

O desempenho em custos foi modelado por meio de uma função fuzzy $f: \mathbb{R}^{6} \rightarrow \mathbb{R}$ tal que $\forall(\tilde{x}, \widetilde{z}) \in \mathbb{R}^{6}=$ $\mathbb{R}^{5} \times \mathbb{R}$,obtém-se o valor do desempenho em custos $\tilde{w}=\tilde{f}(\tilde{y}, \tilde{z})$, em que $\tilde{y}$ é uma variável fuzzy, obtida a partir dos componentes do custo total da cadeia de suprimentos (Total Cost Supply Chain Management), descrita pela função fuzzy $\tilde{\sigma}: \mathbb{R}^{5} \rightarrow \mathbb{R}$, tal que $\tilde{y}=$ $\tilde{g}(x)$. A variável fuzzy $\tilde{z}$ é definida como custo de mercadoria vendida (COGS), e os componentes do

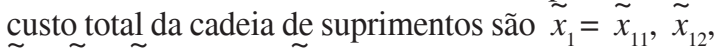
$\tilde{x}_{13}, \tilde{x}_{14}, \tilde{x}_{15}$ em que $\tilde{x}_{11}$ é o custo de planejamento e finanças (Finance and Planning Cost); $\tilde{x}_{12}$, o custo de carregamento de estoques (Inventory Carrying
Cost); $\tilde{x}_{13}$ o custo de tecnologia de informação (IT Cost for Supply Chain); $\tilde{x}_{14}$, o custo de aquisiç̧ão de materiais (Material Acquisition Cost); e $\tilde{x}_{15}$ o custo de gerenciamento do pedido (Order Management Cost), respectivamente.

\subsection{Desempenho da cadeia de suprimentos na gestão de ativos}

O desempenho em ativos foi modelado pela função fuzzy $\tilde{f}: \mathbb{R}^{3} \times \mathbb{R}^{2}=\mathbb{R}^{5} \rightarrow \mathbb{R}$, tal que $\underset{\sim}{\forall}(\tilde{x}, \tilde{z}) \underset{\sim}{\tilde{f}} \in \mathbb{R}^{3} \times$ $\mathbb{R}^{2}$, tem-se o desempenho de ativos $\tilde{w}=\tilde{f}(\tilde{p}, \tilde{q})$, em que as variáveis fuzzy $\tilde{p}$ e $\tilde{q}$ são obtidas a partir dos componentes do ciclo de conversão de caixa (cash to cash) $\left(x_{2}\right)$ e retorno sobre ativos (return over assets) $\left(y_{2}\right)$, respectivamente, e podem ser descritos pelas funções fuzzy $\underset{\sim}{\tilde{g}}: \mathbb{R}^{3} \rightarrow \mathbb{R}$ e $\tilde{h}: \mathbb{R}^{2} \rightarrow \mathbb{R}$, tal que $\forall \tilde{x} \in$ $\mathbb{R}^{3}$ e $\tilde{y} \in \mathbb{R}^{2}, \tilde{p}=\tilde{\sigma}(\underset{\tilde{y}}{\tilde{y}})$ e $q=\tilde{\sim}(y)$. Os componentes das variáveis $\tilde{x}_{2}$ e $\tilde{y}_{2}$ são $\tilde{x}_{21}$ : os dias de contas a receber pendentes (days sales outstanding); $\tilde{x}_{22}$ : dias de suprimento de estoque de uma empresa (inventory days of supply); $\tilde{x}_{23}$ : os dias de contas a pagar pendentes (days payable outstanding); $\tilde{y}_{21}$ : giro de ativos (asset turns), e $\tilde{y}_{22}$ : lucro líquido (net profit), respectivamente.

\subsection{Desempenho da cadeia de suprimentos em confiabilidade}

O desempenho em confiabilidade foi modelado pela função fuzzy $\tilde{f}: \mathbb{R}^{4} \rightarrow \mathbb{R}$, tal que $\forall \tilde{\sim} \in \mathbb{R}^{4}$, tem-se $\widetilde{w}=\tilde{f}(\tilde{z})$ em que a variável fuzzy $\tilde{z}$ é obtida a partir dos componentes do Pedido Perfeito (Perfect Order Fulfillment $)\left(x_{3}\right)$, que pode ser descrito pela função fuzzy $\tilde{g}: \mathbb{R}^{4} \rightarrow \mathbb{R}$, tal que $\tilde{z}=\tilde{g}\left(x_{3}\right)$. Os componentes do pedido perfeito são, respectivamente: $\tilde{x}_{31}$ : confiabilidade quanto à data de entrega (delivery to customer commit date); $\tilde{x}_{32}$ : confiabilidade quanto

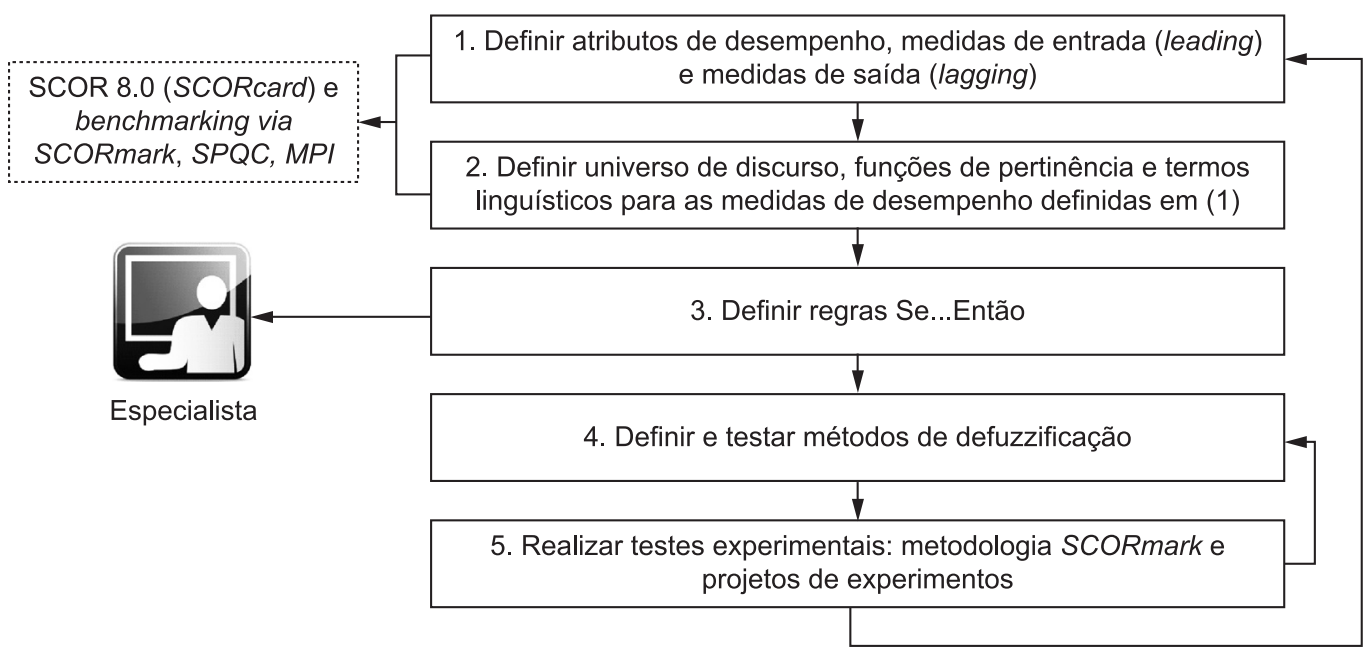

Figura 5. Método de desenvolvimento do modelo. 


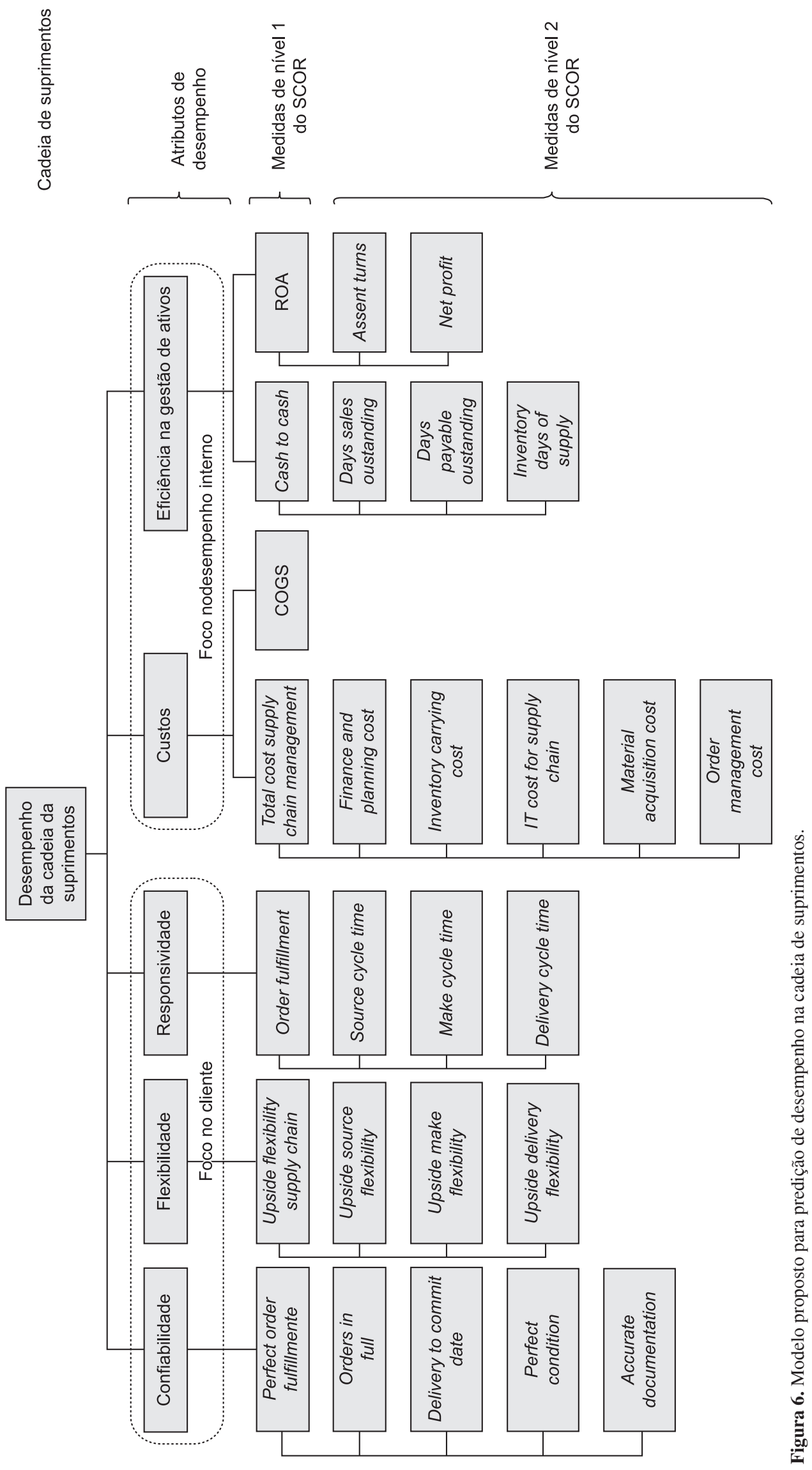


à entrega de pedidos completos (orders delivery in full); $\tilde{x}_{33}$ : confiabilidade quanto à entrega dos produtos livre de avarias (perfect condition); e $\tilde{x}_{34}$ : acurácia na documentação (accurate documentation).

\subsection{Desempenho da cadeia de suprimentos em responsividade}

O desempenho em responsividade foi modelado por meio de uma função fuzzy $\widetilde{f}: \mathbb{R}^{3} \rightarrow \mathbb{R}$, tal que $\forall \tilde{x} \in \mathbb{R}^{3}$, tem-se o desempenho em responsividade $\tilde{w}=\tilde{f}(\tilde{z})$, em que a variável fuzzy $\tilde{z}$ é obtida a partir dos componentes do ciclo de processamento de pedidos (order fulfillment cycle time) $\left(x_{4}\right)$, que pode ser descrito pela função fuzzy $\tilde{g}: \mathbb{R}^{3} \rightarrow \mathbb{R}$, tal que $\tilde{z}=$ $\tilde{g}(x)$. Componentes do order fulfillment cycle time são, respectivamente: $\tilde{x}_{41}$; tempo de ciclo do processo suprimentos (source cycle time); $\tilde{x}_{42}$ : tempo de ciclo do processo de fabricação (make cycle time); e $\tilde{x}_{43}$ : tempo de ciclo do processo de distribuição (delivery cycle time).

\subsection{Desempenho da cadeia de suprimentos em flexibilidade}

O desempenho em flexibilidade foi modelado por meio de uma função fuzzy $\tilde{f}: \mathbb{R}^{4} \rightarrow \mathbb{R}$, tal que $\forall(\tilde{x}$, $\tilde{z}) \in \mathbb{R}^{4}=\mathbb{R}^{3} \times \mathbb{R}$, obtém-se o valor do desempenho em flexibilidade $\tilde{w}=\tilde{f}(\tilde{y}, \tilde{z})$, em que a variável fuzzy $\tilde{y}_{3}$ é obtida a partir dos componentes do incremento de flexibilidade na cadeia de suprimentos (upside supply chain flexibility), sendo descrita pela função fuzzy $\tilde{g}: \mathbb{R}^{3} \rightarrow \mathbb{R}$, tal que $\tilde{y}=\tilde{g}(x)$. A variável fuzzy $\tilde{z}$ é definida como overlapp e os componentes do upside supply chain flexibility são $\tilde{y}_{3}=\tilde{y}_{31}, \quad \tilde{y}_{32}, \tilde{y}_{33}$, em que $\tilde{y}_{31}$ é o tempo necessário para o incremento de flexibilidade do processo de suprimentos (upside source flexibility); $\tilde{y}_{32}$, o tempo necessário para o incremento de flexibilidade do processo de fabricação (upside make flexibility); e ${ }_{3}$, o tempo necessário para o incremento de flexibilidade do processo de entrega (upside delivery flexibility), respectivamente.

Passo 2: Definição do universo de discurso (valores mínimos e máximos), funções de pertinência e termos linguísticos:

A parametrização do universo de discurso das variáveis foi baseada em informações de benchmarking de inúmeras fontes, tais como o Supply Chain Council, Productivity and Quality with Performance Measures \& Metrics (APQC) e o Manufacturing Performance Institute (MPI).

Neste trabalho, foram utilizadas, predominantemente, funções de pertinência triangulares. A Tabela 2 ilustra uma amostra das funções de pertinência para as variáveis de entrada, ou seja, as métricas de nível 2 do SCOR. As funções de pertinência estão descritas segundo o formato proposto na seção 3.1 .

Passo 3: As regras de inferência foram definidas baseadas nos relacionamentos causais propostos pelo SCORcard (SCOR, 2006). Os termos linguísticos (por exemplo: muito baixo, baixo, médio, alto e muito alto) das métricas de resposta (lagging) foram definidas pelos autores (atuando como especialistas) em função das métricas de entrada (leading). Por exemplo, na primeira linha da Tabela 3 , a métrica de resposta gestão de ativos foi definida como "baixa" quando os níveis das métricas de entrada cash to cash e retorno sobre ativos eram respectivamente "melhor" e "muito baixo", respectivamente.

Passo 4: Os métodos de defuzzificação foram definidos de maneira empírica, avaliando principalmente o conceito de continuidade e descontinuidade na fuzzificação. Nesse sentido, foram utilizados (na maioria dos casos) os métodos de defuzzificação CoM e CoA, por serem considerados métodos contínuos. O método CoM é mais indicado no suporte a decisões quantitativas, como é o caso desta pesquisa. A Tabela 4 traz uma amostra das funções de pertinência para as variáveis de resposta e os métodos de defuzzificação utilizados.

Passo 5: O modelo foi testado utilizando-se de números aleatórios e adotando-se as categorias de desempenho propostas pelo SCORmark (superior, vantagem, paridade) (FRANCIS, 2009). Foi testado também o efeito das medidas de entrada (leading) sobre as medidas de saída (lagging). Tal estudo foi realizado por meio de técnicas de projetos de experimentos (DoE), mais particularmente, análises por superfície de resposta, discutido com maiores detalhes na seção 6 .

\section{Resultados}

Com o propósito de analisar o comportamento de resposta gerado pelo modelo, dados experimentais foram simulados por meio da geração de números aleatórios das métricas de nível 2 do modelo SCOR (métrica de entrada - leading). Para tanto, os números aleatórios foram gerados utilizando-se da expressão 8 , por meio do método da transformação inversa, para uma distribuição contínua uniforme.

$$
\chi \leftarrow[a+(b-a) \times \operatorname{random}(s)]
$$

O intervalo de variação (valores máximos e mínimos) para a geração dos números aleatórios das métricas leading foi baseado em inúmeras fontes como o Supply Chain Council, Productivity and Quality with Performance Measures \& Metrics (APQC) e o Manufacturing Performance Institute. 
Tabela 2. Amostra de funções de pertinência e termos linguísticos das variáveis de entrada.

\begin{tabular}{|c|c|c|c|c|}
\hline \multirow{2}{*}{$\begin{array}{l}\text { Métricas } \\
\text { de nível } 1\end{array}$} & \multirow[t]{2}{*}{ Unidade } & \multicolumn{3}{|c|}{ Funções de pertinência triangulares e termos linguísticos } \\
\hline & & baixo & médio & alto \\
\hline Accurate documentation & $\%$ & $(84 ; 1)(91 ; 0)$ & $(84 ; 0)(91 ; 1)(98 ; 0)$ & $(91 ; 0)(98 ; 1)$ \\
\hline Delivery commit date & $\%$ & $(85 ; 1)(91,5 ; 0)$ & $(85 ; 0)(91,5 ; 1)(98 ; 0)$ & $(91,5 ; 0)(98 ; 1)$ \\
\hline Orders in full & $\%$ & $(85 ; 1)(91,5 ; 0)$ & $(85 ; 0)(91,5 ; 1)(98 ; 0)$ & $(91,5 ; 0)(98 ; 1)$ \\
\hline Perfect condition & $\%$ & $(85 ; 1)(91,5 ; 0)$ & $(85 ; 0)(91,5 ; 1)(98 ; 0)$ & $(91,5 ; 0)(98 ; 1)$ \\
\hline Asset turns & giros & $(0,1 ; 1)(1,55 ; 0)$ & $(0,1 ; 0)(1,55 ; 1)(3 ; 0)$ & $(1,55 ; 0)(3 ; 1)$ \\
\hline Net profit & $\%$ da receita & $(0,1 ; 1)(3,7 ; 0)$ & $(0,1 ; 0)(3,7 ; 1)(7,3 ; 0)$ & $(3,7 ; 0)(7,3 ; 1)$ \\
\hline Inventory days of supply & dias & $(27,6 ; 1)(53,8 ; 0)$ & $(27,6 ; 0)(51,9 ; 1)(80 ; 0)$ & $(53,8 ; 0)(80 ; 1)$ \\
\hline Days payable outstanding & dias & $(30 ; 1)(51 ; 0)$ & $(30 ; 0)(51 ; 1)(72 ; 0)$ & $(51 ; 0)(72 ; 1)$ \\
\hline Days sales outstanding & dias & $(25 ; 1)(47,5 ; 0)$ & $(27 ; 0)(47,5 ; 1)(70 ; 0)$ & $(47,5 ; 0)(70 ; 1)$ \\
\hline Delivery cycle time & dias & $(1 ; 1)(4 ; 0)$ & $(1 ; 0)(4 ; 1)(7 ; 0)$ & $(4 ; 0)(7 ; 1)$ \\
\hline Make cycle time & dias & $(1 ; 1)(4 ; 0)$ & $(1 ; 0)(4 ; 1)(7 ; 0)$ & $(4 ; 0)(7 ; 1)$ \\
\hline Source cycle time & dias & $(1 ; 1)(3,5 ; 0)$ & $(1 ; 0)(3,5 ; 1)(6 ; 0)$ & $(3,5 ; 0)(6 ; 1)$ \\
\hline Upside deliver flexibility & dias & $(10 ; 1)(45 ; 0)$ & $(10 ; 0)(45 ; 1)(80 ; 0)$ & $(45 ; 0)(80 ; 1)$ \\
\hline Upside make flexibility & dias & $(10 ; 1)(45 ; 0)$ & $(10 ; 0)(45 ; 1)(80 ; 0)$ & $(45 ; 0)(80 ; 1)$ \\
\hline Upside sorce flexibility & dias & $(10 ; 1)(45 ; 0)$ & $(10 ; 0)(45 ; 1)(80 ; 0)$ & $(45 ; 0)(80 ; 1)$ \\
\hline Finance e plannig cost & $\%$ da receita & $(0,8 ; 1)(1,3 ; 0)$ & $(0,8 ; 0)(1,3 ; 1)(1,8 ; 0)$ & $(1,3 ; 0)(1,8 ; 1)$ \\
\hline Inventory carrying cost & $\%$ da receita & $(2,6 ; 1)(4,15 ; 0)$ & $(2,6 ; 0)(4,15 ; 1)(5,7 ; 0)$ & $(4,15 ; 0)(5,7 ; 1)$ \\
\hline IT cost for supply chain & $\%$ da receita & $(1,7 ; 1)(2,7 ; 0)$ & $(1,7 ; 0)(2,7 ; 1)(3,7 ; 0)$ & $(2,7 ; 0)(3,7 ; 1)$ \\
\hline Material acquisition cost & $\%$ da receita & $(5,2 ; 1)(8,3 ; 0)$ & $(5,2 ; 0)(8,3 ; 1)(11,4 ; 0)$ & $(8,3 ; 0)(11,4 ; 1)$ \\
\hline Order management cost & $\%$ da receita & $(8,2 ; 1)(13,1 ; 0)$ & $(8,2 ; 0)(13,1 ; 1)(18 ; 0)$ & $(13,1 ; 0)(18 ; 1)$ \\
\hline Cost of goods sold & $\%$ da receita & $(49 ; 1)(59,5 ; 0)$ & $(49 ; 0)(59,5 ; 1)(70 ; 0)$ & $(59,5 ; 0)(70 ; 1)$ \\
\hline
\end{tabular}

A mesma classificação utilizada pelo Supply Chain Council (2009) para comparar padrões de benchmarking, denominada Supply Chain SCORmark, foi adotada para analisar os resultados. Tal abordagem estratifica o desempenho de acordo com três posicionamentos:

- Superior: é o desempenho (mediana) de um determinado indicador em relação a um percentual de $10 \%$ das cadeias de suprimentos mais bem classificadas em relação ao total de SCs pesquisadas.

- Vantagem: é o desempenho do ponto médio entre as empresas Top 10 e a mediana de todas as cadeias de suprimentos estudadas.

- Paridade: é o desempenho (mediana) de todas as cadeias de suprimentos estudadas.

O objetivo foi analisar as fronteiras do modelo proposto. Desta maneira foram utilizadas apenas as categorias superior e paridade. A Tabela 5 exibe os resultados destas simulações para as variáveis de resposta (lagging). Os resultados obtidos com o modelo apresentaram-se consistentes quando comparados aos índices de desempenho provenientes das fontes de benchmarking do SCOR, sugerindo que o modelo fuzzy se mostrou satisfatório na simulação do processo de predição de desempenho da cadeia de suprimentos.

\section{Análise estatística das respostas geradas pelo modelo de predição $f u z z y$}

A fim de se compreender o efeito da base de regras sobre as relações de causa e efeito entre as variáveis de entrada (leading) e as variáveis de resposta (lagging), este trabalho utilizou a Metodologia da Superfície de Resposta (RSM), uma técnica de planejamento e análise de experimentos - DoE (MONTGOMERY, 2005). A RSM é um conjunto de técnicas de planejamento e análise de experimentos utilizado na modelagem matemática de uma resposta influenciada por inúmeras variáveis independentes e cujo objetivo é otimizar a resposta (MONTGOMERY, 2005). Uma técnica de planejamento usada é o arranjo do composto central (Central Composite Design $\mathrm{CCD}$ ), que usa uma combinação de arranjos fatoriais (completos ou fracionados), pontos médios dos fatores nivelados (pontos centrais) e os pontos axiais (extremos) para ajustar aos resultados do experimento, quando necessário, um modelo polinomial de segunda ordem (MONTGOMERY, 2005).

Como exemplo ilustrativo da aplicação da RSM para analisar a resposta gerada pelo modelo de simulação, adota-se a variável resposta (lagging) cash to cash. 
Tabela 3. Amostra do bloco de regras para desempenho em ativos.

\begin{tabular}{|c|c|c|}
\hline \multicolumn{2}{|c|}{ Se } & \multirow{2}{*}{$\begin{array}{c}\text { Então } \\
\text { Desempenho } \\
\text { em ativos }\end{array}$} \\
\hline $\begin{array}{c}\text { Cash to } \\
\text { cash }\end{array}$ & $\begin{array}{c}\text { Retorno } \\
\text { sobre ativos }\end{array}$ & \\
\hline Melhor & Muito Baixo & Baixo \\
\hline Melhor & Baixo & Médio \\
\hline Melhor & Médio & Alto \\
\hline Melhor & Alto & Muito Alto \\
\hline Melhor & Muito Alto & Muito Alto \\
\hline Bom & Muito Baixo & Médio \\
\hline Bom & Baixo & Médio \\
\hline Bom & Médio & Médio \\
\hline Bom & Alto & Alto \\
\hline Bom & Muito Alto & Muito Alto \\
\hline Médio & Muito Baixo & Muito Baixo \\
\hline Médio & Baixo & Baixo \\
\hline Médio & Médio & Médio \\
\hline Médio & Alto & Médio \\
\hline Médio & Muito Alto & Alto \\
\hline Ruim & Muito Baixo & Muito Baixo \\
\hline Ruim & Baixo & Baixo \\
\hline Ruim & Médio & Baixo \\
\hline Ruim & Alto & Médio \\
\hline Ruim & Muito Alto & Médio \\
\hline Pior & Muito Baixo & Muito Baixo \\
\hline Pior & Baixo & Muito Baixo \\
\hline Pior & Médio & Baixo \\
\hline Pior & Alto & Médio \\
\hline Pior & Muito Alto & Alto \\
\hline
\end{tabular}

O conceito de ciclo de conversão de caixa ( cash to cash) representa uma medida do uso eficaz do caixa de uma organização. Embora o estoque geralmente seja registrado como um ativo circulante no balanço patrimonial, o valor monetário pode não ser um indicador válido da distribuição real de ativos da organização. Uma parte do estoque pode ter sido entregue a clientes que, devido às condições de crédito da venda, ainda não pagaram as faturas correspondentes. Por outro lado, uma empresa pode dever aos seus fornecedores por produtos e componentes que não estão em sua propriedade. Portanto, a duração do ciclo de conversão de caixa é o tempo necessário para se converter, por exemplo, $\mathrm{R} \$ 1,00$ gasto em estoque em $\mathrm{R} \$ 1,00$ recebido de receita de vendas (BOWERSOX; CLOSS; COOPER, 2007).

Nesse sentido, para avaliar o efeito das métricas Inventory Days of Supply, Days Payable Outstanding e Days Sales Outstanding, sobre Cash to Cash, foi realizado um planejamento composto central $\left(2^{3}\right)$, constituído por oito ensaios lineares nos níveis -1 e +1 , seis ensaios axiais $(\alpha=1,68179)$ e dois ensaios no ponto central, como pode ser observado na Tabela 6 .

A Tabela 7 apresenta os resultados da análise de variância (ANOVA) para $\mathrm{p} \leq 0,05$.

Analisando a Tabela 7, percebe-se que as métricas Inventory days of supply e Days Sales Outstanding apresentaram significância $(\mathrm{p}<0,05)$ para um modelo de ajuste linear, enquanto a métrica Days Payable Outstanding apresentou significância tanto para um modelo linear quanto para um modelo quadrático.

A adequação do modelo à resposta verdadeira exige, portanto, a avaliação correta dos resíduos: a representação da probabilidade normal em função dos resíduos deve ser aproximadamente linear, e a representação da função resíduos, em função da

Tabela 4. Amostra de funções de pertinência e métodos de defuzzificação de medidas lagging.

\begin{tabular}{|c|c|c|c|c|c|c|c|}
\hline \multirow{2}{*}{$\begin{array}{l}\text { Variável } \\
\text { resposta }\end{array}$} & \multirow[t]{2}{*}{ Unidade } & \multirow{2}{*}{$\begin{array}{c}\text { Método de } \\
\text { defuzzificação }\end{array}$} & \multicolumn{5}{|c|}{ Funções de pertinência e termos linguísticos } \\
\hline & & & $\begin{array}{l}\text { Muito } \\
\text { baixo }\end{array}$ & Baixo & Médio & Alto & $\begin{array}{c}\text { Muito } \\
\text { alto }\end{array}$ \\
\hline $\begin{array}{l}\text { Perfect order } \\
\text { fulfillment }\end{array}$ & $\%$ & $\mathrm{CoM}$ & $\begin{array}{c}(85 ; 0) \\
(89,3 ; 1)\end{array}$ & $\begin{array}{l}(87,2 ; 0) \\
(89,3 ; 1) \\
(91,5 ; 0)\end{array}$ & $\begin{array}{l}(89,3 ; 0) \\
(91,5 ; 1) \\
(93,6 ; 0)\end{array}$ & $\begin{array}{c}(91,5 ; 0) \\
(93,6 ; 1) \\
(95,8 ; 0)\end{array}$ & $\begin{array}{r}(93,6 ; 0) \\
(98 ; 1)\end{array}$ \\
\hline $\begin{array}{l}\text { Reliability } \\
\text { supply chain }\end{array}$ & $\%$ & $\mathrm{CoM}$ & $\begin{array}{c}(0 ; 0) \\
(33,3 ; 1)\end{array}$ & $\begin{array}{c}(87,2 ; 0) \\
(89,3 ; 1) \\
(91,5 ; 0)\end{array}$ & $\begin{array}{c}(89,3 ; 0) \\
(91,5 ; 1) \\
(93,6 ; 0)\end{array}$ & $\begin{array}{l}(91,5 ; 0) \\
(93,6 ; 1) \\
(95,8 ; 0)\end{array}$ & $\begin{array}{r}(93,6 ; 0) \\
(98 ; 1)\end{array}$ \\
\hline $\begin{array}{l}\text { Return over } \\
\text { assets (ROA) }\end{array}$ & $\begin{array}{l}\% \text { da } \\
\text { receita }\end{array}$ & $\mathrm{CoA}$ & $\begin{array}{c}(0,01 ; 0) \\
(7,3 ; 1)\end{array}$ & $\begin{array}{l}(3,7 ; 0) \\
(7,3 ; 1) \\
(11 ; 0)\end{array}$ & $\begin{array}{c}(7,3 ; 0) \\
(11 ; 1) \\
(14,7 ; 0)\end{array}$ & $\begin{array}{c}(11 ; 0) \\
(14,7 ; 1) \\
(18,3 ; 0)\end{array}$ & $\begin{array}{r}(14,7 ; 0) \\
(22 ; 1)\end{array}$ \\
\hline $\begin{array}{l}\text { Total } \\
\text { SCM cost }\end{array}$ & $\begin{array}{l}\% \text { da } \\
\text { receita }\end{array}$ & $\mathrm{CoM}$ & $\begin{array}{l}(24 ; 0) \\
(27,7 ; 1)\end{array}$ & $\begin{array}{l}(25,8 ; 0) \\
(27,7 ; 1) \\
(29,6 ; 0)\end{array}$ & $\begin{array}{l}(27,7 ; 0) \\
(29,6 ; 1) \\
(31,4 ; 0)\end{array}$ & $\begin{array}{l}(29,5 ; 0) \\
(31,4 ; 1) \\
(33,2 ; 0)\end{array}$ & $\begin{array}{l}(31,4 ; 0) \\
(35,1 ; 1)\end{array}$ \\
\hline
\end{tabular}




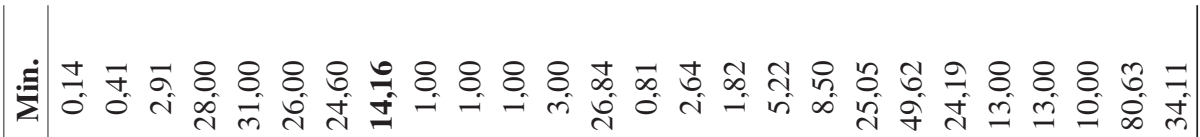

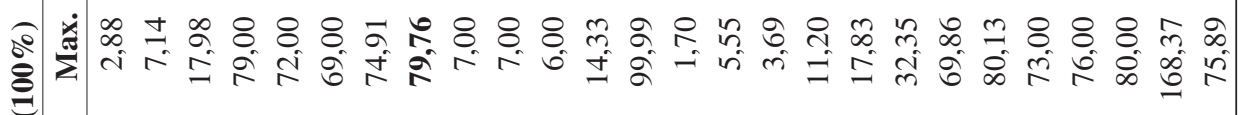

भ $\infty$ t

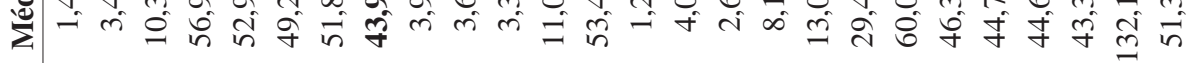

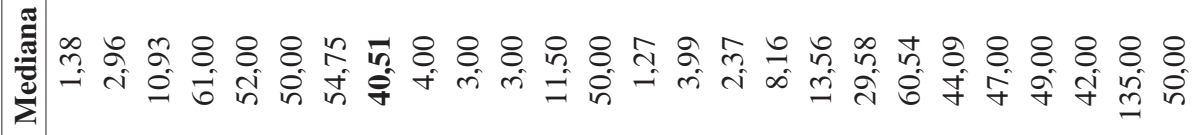
$\stackrel{-3}{3}$

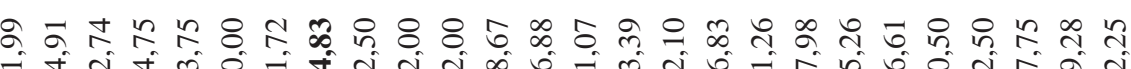

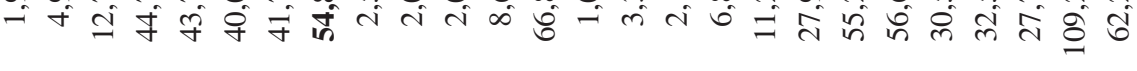

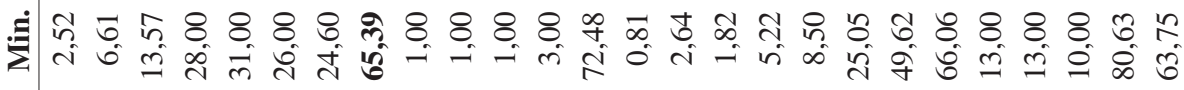

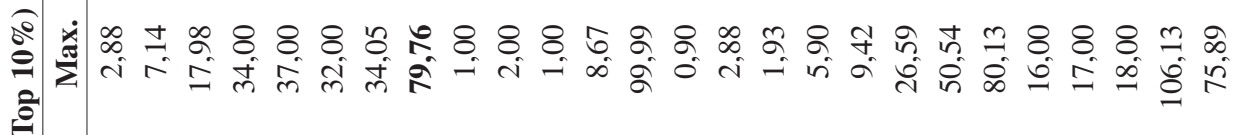

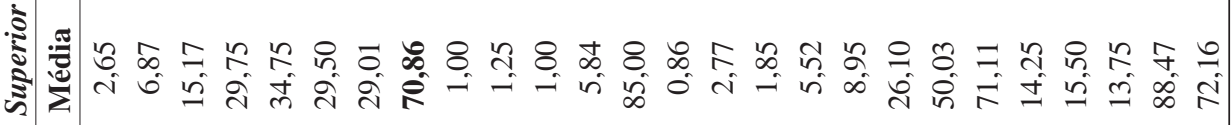

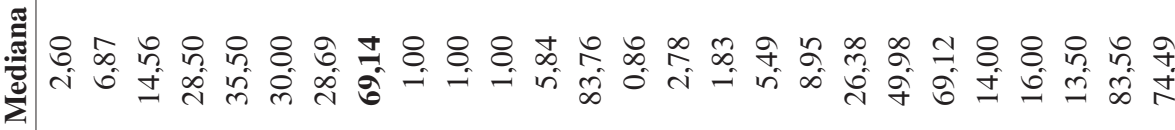

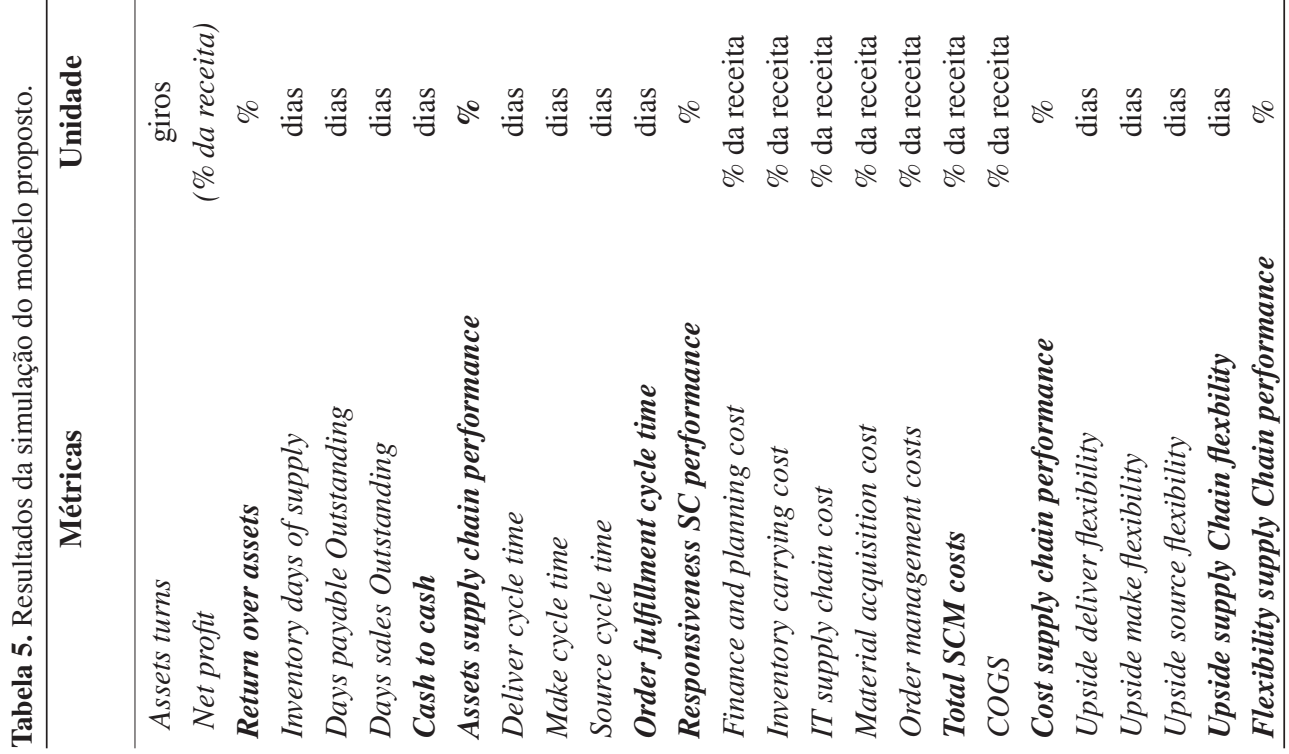


Tabela 6. Planejamento experimental composto central para cash to cash.

\begin{tabular}{|c|c|c|c|c|c|c|c|}
\hline \multirow[t]{2}{*}{ Ensaios } & \multicolumn{3}{|c|}{ Não Codificado } & \multicolumn{3}{|c|}{ Codificado } & \multirow{2}{*}{$\begin{array}{c}\text { Resposta } \\
\left(\mathbf{x}_{2}\right)\end{array}$} \\
\hline & $\sim x_{22}$ & $\sim x_{23}$ & $\sim x_{21}$ & $\sim x_{22}$ & $\sim x_{23}$ & $\sim x_{21}$ & \\
\hline 1 & 42,80 & 40,00 & 34,50 & $-1,00000$ & $-1,00000$ & $-1,00000$ & 47,93 \\
\hline 2 & 42,80 & 40,00 & 60,50 & $-1,00000$ & $-1,00000$ & 1,00000 & 58,46 \\
\hline 3 & 42,80 & 62,00 & 34,50 & $-1,00000$ & 1,00000 & $-1,00000$ & 36,97 \\
\hline 4 & 42,80 & 62,00 & 60,50 & $-1,00000$ & 1,00000 & 1,00000 & 44,25 \\
\hline 5 & 64,80 & 40,00 & 34,50 & 1,00000 & $-1,00000$ & $-1,00000$ & 56,36 \\
\hline 6 & 64,80 & 40,00 & 60,50 & 1,00000 & $-1,00000$ & 1,00000 & 63,6 \\
\hline 7 & 64,80 & 62,00 & 34,50 & 1,00000 & 1,00000 & $-1,00000$ & 42,13 \\
\hline 8 & 64,80 & 62,00 & 60,50 & 1,00000 & 1,00000 & 1,00000 & 61,57 \\
\hline 9 & 35,30 & 51,00 & 47,50 & $-1,68179$ & 0,00000 & 0,00000 & 43,93 \\
\hline 10 & 72,30 & 51,00 & 47,50 & 1,68179 & 0,00000 & 0,00000 & 56,93 \\
\hline 11 & 53,80 & 32,50 & 47,50 & 0,00000 & $-1,68179$ & 0,00000 & 74,67 \\
\hline 12 & 53,80 & 69,50 & 47,50 & 0,00000 & 1,68179 & 0,00000 & 50,3 \\
\hline 13 & 53,80 & 51,00 & 25,64 & 0,00000 & 0,00000 & $-1,68179$ & 41,34 \\
\hline 14 & 53,80 & 51,00 & 69,36 & 0,00000 & 0,00000 & 1,68179 & 59,29 \\
\hline $15(\mathrm{C})$ & 53,80 & 51,00 & 47,50 & 0,00000 & 0,00000 & 0,00000 & 50,3 \\
\hline $16(\mathrm{C})$ & 53,80 & 51,00 & 47,50 & 0,00000 & 0,00000 & 0,00000 & 50,3 \\
\hline
\end{tabular}

Em que: $\left(\tilde{x}_{22}\right)$ inventory days of supply (dias); $\left(\tilde{x}_{23}\right)$ days payable outstanding (dias); $\left(\tilde{x}_{21}\right)$ days sales outstanding (dias); $\left(\mathrm{x}_{2}\right)$ cash to cash (resposta) (dias).

Tabela 7. ANOVA para cash to cash $\left(\mathrm{R}^{2}=0,92557\right.$; Adj: 0,87596).

\begin{tabular}{lrrrrl}
\hline \multicolumn{1}{c}{ Fatores } & SS & df & MS & F & P ( $\leq \mathbf{0 , 0 5 )}$ \\
\hline Inventory days of supply $(L)$ & 245,587 & 1 & 245,5871 & 20,29740 & 0,001478 \\
Inventory days of supply $(Q)$ & 4,687 & 1 & 4,6868 & 0,38735 & 0,549135 \\
Days payable outstanding $(L)$ & 497,354 & 1 & 497,3545 & 41,10558 & 0,000124 \\
Days payable outstanding $(Q)$ & 116,811 & 1 & 116,8105 & 9,65421 & 0,012577 \\
Days sales outstanding $(L)$ & 408,355 & 1 & 408,3552 & 33,74993 & 0,000256 \\
Days sales outstanding $(Q)$ & 5,241 & 1 & 5,2414 & 0,43319 & 0,526902 \\
Erro & 108,895 & 9 & 12,0994 & & \\
Total SS & 1463,135 & 15 & & & \\
\hline
\end{tabular}

resposta prevista, deve ser aleatória e razoavelmente simétrica (MYERS; MONTGOMERY, 2002). A análise do gráfico da distribuição normal dos resíduos para o modelo Cash to Cash sugere que resíduos podem ser julgados como normalmente distribuídos, e, portanto, a hipótese de normalidade da resposta está satisfeita.

Em alguns casos, a visualização do comportamento da superfície de resposta é complexa e limitada. Para tanto, é utilizada uma projeção dessa superfície de resposta, denominada curva de nível. A análise por meio dessas linhas de contorno possibilita uma leitura gráfica na busca do ponto que dê a resposta ótima, que maximiza os resultados do sistema. A Figura 7 ilustra uma curva de nível para o modelo cash to cash.

Analisando a medida cash to cash em função das variáveis Days payable outstanding e Days sales outstanding (Figura 7), percebeu-se um tempo de conversão de caixa "ótimo" para um valor abaixo de 32 dias. O ponto ótimo ocorreu quando as métricas Days sales outstanding e days payable outstanding desempenharam 20 e 63 dias, respectivamente. Tal desempenho ilustra perfeitamente a influência das duas variáveis com maiores efeitos no modelo, ou seja, Days sales outstanding e days payable outstanding.

O mesmo procedimento de experimentação e análise dos resultados foi realizado para as outras métricas de resposta (métricas de nível 1 do modelo SCOR indicadas na Figura 6). A Tabela 8 apresenta o nível de significância (valor de p) para um ajustamento linear das cinco métricas de nível 1 . Observando os dados, constata-se que a maior parte das métricas de entrada (leading) revelou ter um efeito significativo sobre as métricas de resposta (lagging), com p <0,05. 
Tabela 8. P-valor da ANOVA das métricas de entrada e saída do modelo SCOR.

\begin{tabular}{lcc}
\hline Métricas de saída (Lagging) & Métricas de entrada (Leading) & $\boldsymbol{P}$ value $(<\mathbf{0 , 0 5})$ \\
\hline Perfect order fulfillment & Accurate documentation & $\mathbf{0 , 0 0 0 2 0 2}$ \\
& Delivery to commit date & $\mathbf{0 0 0 3 7 2}$ \\
Order fulfillment cycle time & Orders in full & $\mathbf{0 0 0 1 1 2}$ \\
& Perfect condition & $\mathbf{0 , 0 0 0 1 1 1}$ \\
Return over assets & Delivery cycle time & $\mathbf{0 , 0 0 8 9 8 3}$ \\
& Make cycle time & $\mathbf{0 , 0 0 8 9 8 3}$ \\
Upside flexibility supply chain & Source cycle time & 0,229607 \\
& Asset turns & $\mathbf{0 , 0 0 0 0 2 1}$ \\
Total cost supply chain management & Net profit & $\mathbf{0 , 0 0 0 0 1 9}$ \\
& Upside source flexibility & 0,070937 \\
& Upside make flexibility & 0,070624 \\
& Upside deliver flexibility & 0,070624 \\
& Finance and planning cost & 0,428424
\end{tabular}

Fitted surface; Variable: cash to cash 3 factors, 1 blocks, 16 runs; MS residual = 13,18734 DV: cash to cash

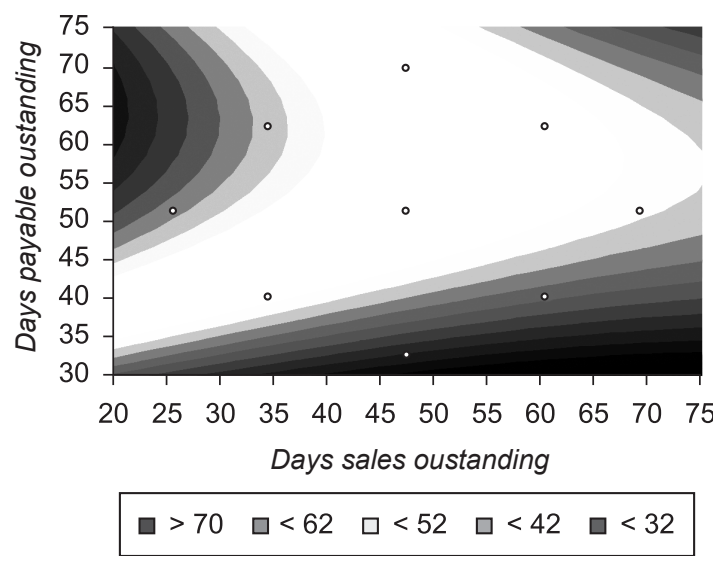

Figura 7. Cash to Cash (dias) em função de Days sales outstanding (dias) e Days payable outstanding (dias).

\section{Conclusões}

Embora a literatura sobre avaliação de desempenho em cadeias de suprimentos proponha inúmeras medidas e modelos, a utilização de métricas de desempenho para gerenciar o desempenho de cadeias de suprimentos é uma atividade extremamente complexa devido:

- À existência de uma grande quantidade de métricas;

- À inexistência de dados ou à dificuldade no compartilhamento deles ao longo da cadeia;

- Métodos de comparação pareados sujeitos à erros de julgamento humano;
- à natureza qualitativa de muitas métricas, e;

- Aos numerosos relacionamentos de causa e efeito entre as medidas e a falta de modelagem matemática para quantificar tais relacionamentos.

Todas essas características tornam difícil a predição do desempenho em cadeias de suprimentos. Deste modo, a lógica fuzzy, um tipo de sistema especialista (LIAO, 2005), configura-se como uma abordagem possível para tal propósito, por justamente tratar de situações como as descritas anteriormente. Nesse sentido, diferentemente de outros sistemas especialistas (abordagens heurísticas), a lógica fuzzy aparece como uma boa alternativa de modelagem matemática para predição do desempenho em cadeias de suprimentos (FOGGIN; MENTZER; MONROE, 2004).

O modelo SCOR foi escolhido por incorporar alguns processos de negócios ao longo da cadeia de suprimentos, reforçando a necessidade de se desenvolver uma avaliação de desempenho sistêmica (HOLMBERG, 2000). No entanto, o modelo SCOR não dispõe de ferramentas analíticas para suportar o processo de tomada de decisões estratégicas na gestão da cadeia de suprimentos (HUANG; SHEORAN; WANG, 2004). Abordagens denominadas "Supply Chain Design Management" têm emergido como ferramentas analíticas interessantes (POLUHA, 2007) para auxiliar as empresas na identificação e melhoria dos processos de negócios da cadeia de suprimentos, por meio da simulação e predição do desempenho e decisões estruturais quando sujeitas a diferentes cenários (What-If impact analysis).

O modelo proposto neste trabalho tornou possível predizer o desempenho da cadeia de suprimentos de maneira sistêmica, dado que a lógica de modelagem 
de um sistema fuzzy baseado em regras é baseada numa visão de processos interconectados, que são estruturados por variáveis de entrada (métricas leading), máquina de inferência e variáveis de saída (métricas lagging), conforme idealizado por Unahabhokha, Platts e Tan (2007). Deste modo, se uma variável de entrada (métrica leading) é alterada, o desempenho do sistema como um todo é afetado. Uma contribuição importante deste trabalho foi incluir no modelo analítico, as métricas de nível 2 do modelo SCOR.

Dados aleatórios foram usados para testar o modelo, gerando resultados que se mostraram muito consistentes quando comparados aos scorecards de muitas empresas que usam o SCORmark como um padrão de benchmarking em cadeias de suprimentos. Análises estatísticas dos resultados usando superfície de resposta revelaram que a maior parte das métricas (variáveis de resposta) apresentou um comportamento linear no relacionamento com as métricas de níveis inferiores (variáveis de entrada no modelo). Também, a análise de variância (ANOVA, com $p<0,05$ ) mostrou a significância dos efeitos da maior parte das métricas sobre as respostas, confirmando a relevância dos relacionamentos causais incorporados no modelo.

Portanto, a principal contribuição da pesquisa descrita neste trabalho foi a proposição de uma ferramenta analítica para gestão de cadeias de suprimentos baseada nas métricas de nível um e dois do modelo SCOR, capaz de predizer o desempenho de uma cadeia de suprimentos baseado em análises de impacto "O que aconteceria se...".

Em geral, os resultados reforçam a proposição de que a adoção de um modelo de predição baseado em lógica fuzzy e nas métricas do SCOR constitui uma abordagem razoável para predizer o desempenho de cadeias de suprimentos, auxiliando desta maneira os gerentes no processo de tomada de decisão do gerenciamento do desempenho dessas cadeias de suprimentos. O próximo passo desta pesquisa é validar o modelo com dados reais. Além disso, seria interessante customizar o modelo com as métricas de uma determinada cadeia de suprimentos, bem como modelar a base de regras de acordo com a visão dos gerentes de supply chain da empresa em questão.

\section{Referências}

AGARWAL, A.; SHANKAR, R.;TIWARI, M. K. Modeling the metrics of lean, agile and leagile supply chain: An ANP-based approach. European Journal of Operational Research, v. 173, p. 211-225, 2006. http://dx.doi. org/10.1016/j.ejor.2004.12.005

AISSAOUI, N.; HAOUARI, M.; HASSINI, E. Supplier selection and order lot sizing modeling: a review. Computers \& Operations Research, v. 34, p. 351640, 2007. http://dx.doi.org/10.1016/j.cor.2006.01.016
ANGERHOFER, B. J.; ANGELIDES, M. C. A model and a performance measurement system for collaborative supply chains. Decision Support Systems, v. 42, p. 283-301, 2006. http://dx.doi.org/10.1016/j. dss.2004.12.005

BARROS, L. C.; BASSANEZI, R. C. Tópicos de lógica fuzzy e biomatemática. Campinas: Comissão de Publicações IMECC/UNICAMP, 2006. (Coleção IMECC, Textos didáticos, v. 5).

BEAMON, B. M. Measuring supply chain performance. International Journal of Operations \& Production Management, v. 19, n. 3, p. 275-292, 1999. http:// dx.doi.org/10.1108/01443579910249714

BEAMON, B. M.; CHEN, V. C. P. Performance analysis of conjoined supply chains. International Journal of Production Research, v. 39, n. 14, p. 3195-218, 2001. http://dx.doi.org/10.1080/00207540110053156

BELTON, V.; STEWART, T. J. Multiple Criteria Decision Analysis: An Integrated Approach. Boston: Kluwer Academic Publishers, 2002.

BERTRAND, J. W. M.; FRANSOO, J. Operations management research methodologies using quantitative modeling. International Journal of Operations and Production Management, v. 22, n. 2, p. 241-264, 2002. http://dx.doi.org/10.1108/01443570210414338

BLINGE, M.; SWENSSON, A. Working with Heavy EcoDriving. Stockholm: Carlsberg Sverige AB, 2006.

BOJADZIEV, G.; BOJADZIEV, M. Fuzzy logic for business, finance, and management. Danvers: World Scientific, 1997. (Advances in Fuzzy Systems - Applications and Theor, v.12).

BOLSTORFF, P. Measuring the impact of supply chain performance. CLO - Chief Logistics Officers, v. 12, p. 5-11, 2003.

BOLSTORFF, P.; ROSENBAUM, R. Supply chain excelence. A handbook for dramatic improvement using the SCOR Model. New York: AMACOM, 2007.

BOURNE, M. et al. Designing, implementing and updating performance measurement systems. International Journal of Operations \& Production Management, v. 20, v. 7, p. 754-71, 2000.

BOURNE, M. et al. The success and failure of performance measurement initiatives: perceptions of participating managers. International Journal of Operations \& Production Management, v. 22, v. 11, p. 1288-310, 2002.

BOWERSOX, D. J.; CLOSS, D. J.; COOPER, M. B. Gestão da cadeia de suprimentos e logística. Rio de Janeiro: Elsevier, 2007.

BREWER, P. C.; SPEH, T. W. Using the Balanced Scorecard to measure supply chain performance. Journal of Business Logistics, v. 211, p. 75-93, 2000.

BRINDLEY, C.; RITCHIE, B. Supply chain risk management and performance: a guiding framework for future development. International Journal of Operations \& Production Management, v. 27, n. 3, p. 303-322, 2007. http://dx.doi.org/10.1108/01443570710725563

BULLINGER, H. J.; KUHNER, M.; VAN HOFF, A. Analysing supply chain performance using a balanced measurement method. International Journal of 
Production Research, v. 4015, p. 3533-43, 2002. http://dx.doi.org/10.1080/00207540210161669

CAI, J.; LIU, X.; LIU, J. Improving supply chain performance management: A systematic approach to analyzing iterative KPI accomplishment. Decision Support Systems, v. 46, p. 512-521, 2009. http://dx.doi. org/10.1016/j.dss.2008.09.004

CARLSSON, C.; FEDRIZZI, M.; FULLÉR, R. Fuzzy logic in management. Massachusetts: Kluwers Academic Publishers, 2004. http://dx.doi. org/10.1007/978-1-4419-8977-2

CHAN, F. T. S. Performance measurement in a supply chain. The International Journal of Advanced Manufacturing Technology, v. 21, p. 534-548, 2003. http://dx.doi.org/10.1007/s001700300063

CHAN, F. T. S. et al. A conceptual model os performance measurement for supply chains. Management Decision, v. 41, n. 7, p. 635-642, 2003. http://dx.doi. org/10.1108/00251740310495568

CHAN, F. T. S.; QI, H. J. An innovative performance measurement method for supply chain management. An International Journal Supply Chain Management, v. 8, n. 3-4, p. 209-223, 2003.

CHANG, R. Y.; MORGAN, M. W. Performance Scorecards: measuring the right things in the real world. San Francisco: Jossey-Bass, 2000. 224 p.

CUTHBERTSON, R.; PIOTROWICZ, W. Supply chain best practices: identification and categorisation of measures and benefits. International Journal of Productivity and Performance Management, v. 57, n. 5, p. 389-404, 2008. http://dx.doi.org/10.1108/17410400810881845

DE TONI, A.; TONCHIA, S. Performance measurement systems: models, characteristics and measures. International Journal of Operations \& Production Management, v. 21, n. 1-2, p. 46-70, 2001. http:// dx.doi.org/10.1108/01443570110358459

DUBOIS, D.; PRADE, H. Fuzzy Sets and Systems. NewYork: Academic Press, 1980.

FOGGIN, J. H.; MENTZER, J. T.; MONROE, C. L. A supply chain diagnostic tool. International Journal of Physical Distribution \& Logistics Management, v. 34, n. 10, p. $827-855,2004$. http://dx.doi. org/10.1108/09600030410571383

FRANCIS, J. Keeping SCORs in Your Supply Chain: Benchmarking. 2009. Disponível em: <http://www. supply-chain.org/benchmarking/presentationS $>$. Acesso em: 15 jun. 2009.

GASPARETTO, V. Proposta de uma sistemática para avaliação de desempenho em cadeias de suprimentos. 2003. 248 f. Tese (Doutorado em Engenharia de Produção)-Universidade Federal de Santa Catarina, Florianópolis, 2003.

GAUDENZI, B.; BORGHESI, A. Managing risks in the supply chain using the AHP method. The International Journal of Logistics Management, v. 17 , n. 1, p. 114-136, 2006. http://dx.doi. org/10.1108/09574090610663464

GOLEÇ, A.; TASKIN, H. Novel methodologies and a comparative study for manufacturing systems performance evaluations. Information Sciences, v. 177, p. 5253-5274, 2007. http://dx.doi.org/10.1016/j. ins.2007.06.024

GUNASEKARAN, A.; PATEL, C.; MCGAUGHEY, R. E. A framework for supply chain performance measurement. International Journal of Production Economics, v. 87, n. 3, p. 333-347, 2004. http://dx.doi. org/10.1016/j.ijpe.2003.08.003

GUNASEKARAN, A.; PATEL, C.; TIRTIROGLU, E. Performance measures and metrics in a supply chain environment. International Journal of Operations \& Production Management, v. 21, n. 1-2, p. 71-87, 2001. http://dx.doi.org/10.1108/01443570110358468

HERVANI, A. A.; HELMS, M. M.; SARKIS, J. Performance measurement for green supply chain management. International Journal Benchmarking, v. 12 , n. 4, p. 330-353, 2005. http://dx.doi. org/10.1108/14635770510609015

HIEBER, R. Supply Chain Management: A Collaborative Performance Measurement Approach. Zurich: VDF, 2002.

HOLMBERG, S. A system perspective on supply chain measurements. International Journal of Physical Distribution \& Logistics Managment, v. 30, n. 10 , p. 847-868, 2000. http://dx.doi. org/10.1108/09600030010351246

HUANG, S. H.; SHEORAN, S. K.; WANG, G. A review and analysis of supply chain operations reference (SCOR) model. An International Journal Supply Chain Management, v. 9, n. 1, p. 23-9, 2004. http:// dx.doi.org/10.1108/13598540410517557

JAIN,V.; TIWARI, M. K.; CHAN, F. T. S. Evaluation of the supplier performance using na evolutionary fuzzy-based approach. Journal of Manufacturing Technology Management, v. 15, n. 8, p. 735-744, 2004. http://dx.doi.org/10.1108/17410380410565320

JESUS, G. P. Estudo de caso sobre a medição de desempenho da cadeia de suprimentos de uma montadora de veículos. 2003. 2157 f. Dissertação (Mestrado em Engenharia de Produção)-Universidade Federal de São Carlos, São Carlos, 2003.

KELTON, W. D.; SADOWSKI, R. P.; SADOWSKI, D. A. Simulation with arena. WCB MacGraw-Hill, 1998. (MacGraw-Hill Series in Industrial Engineering and Management Science).

KENNERLEY, M.; NEELY, A. A framework of the factors affecting the evolution of performance measurement systems. International Journal of Operations \& Production Management, v. 22, n. 11, p. 1222-45, 2002. http://dx.doi.org/10.1108/01443570210450293

KENNERLEY, M.; NEELY, A. Measuring performance in a changing business environment. International Journal of Operations \& Production Management, v. 23, n. 2, p. 213-29, 2003. http://dx.doi. org/10.1108/01443570310458465

KLEIJNEN, J. P. C.; SMITS, M. T. Performance metrics in supply chain management. Journal of the Operational Research Society, v. 54, n. 5, p. 507-514, 2003. http:// dx.doi.org/10.1057/palgrave.jors.2601539

KLIR, G. J.; YUAN, B. Fuzzy sets and Fuzzy logic: theory and applications. Upper Saddle River: Prentice Hall, 1995. 
KUENG, P. Process performance measurement system: a tool to support process-based organizations. Total Quality Management, v. 11, n. 1, p. 67-85, 2000. http://dx.doi.org/10.1080/0954412007035

KUENG, P.; WETTSTEIN, T.; LIST, B. A holistic process performance analysis through a performance data warehouse. In: AMERICAS CONFERENCE ON INFORMATION SYSTEM, 7., 2001, Boston. Proceedings... Boston, 2001. p. 349-356.

LAMBERT, D.; POHLEN, T. Supply chain metrics. International Journal of Logistics Management, v. 12, n. 1, p. 1-19, 2001. http://dx.doi. org/10.1108/09574090110806190

LAPIDE, L. What about measuring supply chain performance? Achieving Supply Chain Excellence through Technology, v. 2, p. 287-297, 2000.

LIAO, S. H. Expert system methodologies and applications: a decade review from 1995 to 2004. Expert Systems with Applications, v. 28, n. 1, p. 93-103, 2005. http:// dx.doi.org/10.1016/j.eswa.2004.08.003

LI, S. et al. Development and validation of a measurement instrument for studying supply chain practices. Journal of Operations Management, v. 23, p. 618-641, 2005.

LIN, C. T.; CHEN, C. T. A fuzzy-logic-based approach for new product Go/NoGo evaluation at the front end. IEEE Transactions on Systems, Man, and Cybernetics Part A, Systems and Humans, v. 34, n. 1, p. 132-142, 2004. http://dx.doi.org/10.1109/TSMCA.2003.818876

LIN, C. T.; CHIU, H.; CHU, P. Y. Agility índex in the supply chain. International Journal Production Economics, v. 100, p. 285-299, 2006. http://dx.doi. org/10.1016/j.ijpe.2004.11.013

LIN, H. Y.; HSU, P. Y.; SHEEN, G. J. A fuzzy-based decision-making procedure for data warehouse system selection. Expert Systems with Applications, v. 32, n. 3, p. 939-953, 2007. http://dx.doi.org/10.1016/j. eswa.2006.01.031

LIU, S. C.; LIU, S. Y. An efficient expert system for air compressor troubleshooting. Expert Systems, v. 18, n. 4, p. 203-214, 2001. http://dx.doi. org/10.1111/1468-0394.00175

LOCKAMY, A.; MCCORMACK, K. Linking SCOR planning practices to supply chain performance: an exploratory study. International Journal of Operations \& Production Management, v. 24, n. 11-12, p. 1192-218, 2004.

MACHACHA, L. L.; BHATTACHARYA, P. A fuzzy logic-based approach to project selection. IEEE Transactions on Engineering Management, v. 47, n. 1, p. 65-73, 2000. http://dx.doi.org/10.1109/17.820726

MON, D. L. ; CHENG, C. H.; LIN, J. C. Evaluating weapon system using fuzzy analytic hierarchy process based on entropy weight. Fuzzy Sets and Systems, v. 62, p. 127-134, 1994. http://dx.doi. org/10.1016/0165-0114(94)90052-3

MONTGOMERY, D. C. Design and Analysis of Experiments: Response surface method and designs. New Jersey: John Wiley and Sons, Inc., 2005.

MORGAN, C. Structure, speed and salience: performance measurement in the supply chain. Business Process Management Journal, v. 10, n. 5, p. 522-36, 2004.
MYERS, R.; MONTGOMERY, D. C. Response Surface Methodology. 2. ed. New York: John Wiley \& Sons, Inc., 2002.

ORDOOBADI, S. Application of Taguchi loss functions for supplier selection. International Journal Supply Chain Management, v. 14, n.1, p. 22-30, 2009. http:// dx.doi.org/10.1108/13598540910927278

PARK, J. H.; LEE, J. K.; YOO, J. S. A framework for designing the balanced supply chain scorecard. European Journal of Information Systems, v. 14, n. 4, p. 335-346, 2005. http://dx.doi.org/10.1057/ palgrave.ejis.3000544

PEDRYCZ, W.; GOMIDE, F. An Introduction to Fuzzy Sets: Analysis and Design. Massachusetts: MIT Press, 1998.

PERIN FILHO, C. Introdução à simulação de sistemas. Campinas: Editora da UNICAMP, 1995.

PIRES, S. R. I. Gestão da cadeia de suprimentos: conceitos, estratégias, práticas e casos. Sao Paulo: Editora Atlas, 2004. 310 p.

PIRES, S. R. I.; ARAVECHIA, C. Measuring supply chain performance. In: ANNUAL CONFERENCE OF THE PRODUCTION AND OPERATIONS MANAGEMENT SOCIETY, 12., 2001, Orlando. Anais... Orlando: 2001.

POLUHA, R. G. Application of the SCOR Model in Supply Chain Management. New York: Cambria Press, 2007.

REINER, G. Supply chain management research methodology using quantitative models based on empirical data. In: KOTZAB, H. et al. (Eds.). Research methodologies in supply chain management. Heidelberg: Physica - Verlag, 2005. http://dx.doi. org/10.1007/3-7908-1636-1_28

ROBSON, S. Real World Research: A resource for social scientists and practitioner researcher. Blackwell Publishers, 2002.

ROSS, T. J. Fuzzy Logic with Engineering Applications. New York: McGraw-Hill, 1997.

SANTOS, S. P.; BELTON, V.; HOWICK, S. Adding value performance measurement by using system dynamics and multicriteria analysis. International Journal of Operations \& Production Management, v. 22, n. 11, p. 1246-1272, 2002. http://dx.doi. org/10.1108/01443570210450284

SANTOS, S. P.; BELTON, V.; HOWICK, S. Enhanced performance measurement using OR: a case study. Journal of the Operational Research Society, v. 59, n. 6, p. 762 - 775, 2008. http://dx.doi.org/10.1057/ palgrave.jors. 2602397

SCHONSLEBEN, P. Integral Logistics Management: Planning and Control of Comprehensive Supply Chains. Boca Raton: St Lucie Press, 2004.

SCOR - Supply Chain Operations Reference Model, Version 8.0, 2006. Supply-Chain Council. Disponível em: /http://www.supply-chain.org/resources/scor/8.0S (Acesso em 3 Jun. 2009).

SELLITTO, M. A.; MENDES, L. W. Avaliação comparativa do desempenho de três cadeias de suprimentos em manufatura. Produção, v. 16, n. 3, p. 552-568, 2006.

SEYDEL, J. Data envelopment analysis for decision support. Industrial Management \& Data 
Systems, v. 106, n. 1, p. 81-96, 2006. http://dx.doi. org/10.1108/02635570610641004

SHARMA, M. K.; BHAGWAT, R. An integrated BSC-AHP approach for supply chain management evaluation. Measuring Business Excellence, v. 11, n. 3, p. 57-68, 2007. http://dx.doi. org/10.1108/13683040710820755

SHEPHERD, C.; GÜNTER, H. Measuring supply chain performance: current research and future directions. International Journal of Productivity and Performance Management, v. 55, n. 3-4, p. 242-258, 2006.

SIMÕES, M. G.; SHAW, I. S. Controle e modelagem fuzzy. 2. ed. São Paulo: Blucher, 2007.

STEPHENS, S. Supply Chain Operations Reference Model 5.0: A New Tool to Improve Supply Chain Efficiency and Achieve Best Practice. International Systems Frontiers, v. 3, n. 4, p. 471-476, 2001. http:// dx.doi.org/10.1023/A:1012881006783

SUPPLY CHAIN COUNCIL - SCC. Supply Chain Operations Reference Model SCOR. Version 8.0. Supply-Chain Council, Inc., 2006. Disponível em: $<$ http://www.supply-chain.org/resources/scor/8.0S >. Acesso em: 3 jun. 2009.

THAKKAR, J. et al. Development of a balanced scorecard: An integrated approach of Interpretive Structural Modeling (ISM) and Analytic Network Process (ANP). International Journal of Productivity and Performance Management, v. 56, n. 1, p. 25-59, 2007. http://dx.doi.org/10.1108/17410400710717073

THEERANUPHATTANA, A.; TANG, J. C. S. A conceptual model of performance measurement for supply chains: Alternative considerations. Journal of Manufacturing Technology Management, v. 19, n. 1, p. 125-148, 2008. http://dx.doi.org/10.1108/17410380810843480

UNAHABHOKHA, C.; PLATTS, K.; TAN, K. H. Predictive performance measurement system. A fuzzy expert system approach. An International Journal Benchmarking, v. 14, n. 1, 2007.

van HOEK, R. I. Measuring the unmeasurable: measuring and improving performance in the supply chain, An International Journal Supply Chain Management, v. 3, n. 4, p. 187-92, 1998. http://dx.doi. org/10.1108/13598549810244232
VARMA, S.; WADHWA S.; DESHMUKH, S. G. Evaluating petroleum supply chain performance: Application of Analytical Hierarchy Process to Balanced Scorecard. Asia Pacific Journal of Marketing \& Logistics, v. 20, n. 3, p. 343-356, 2008. http://dx.doi. org/10.1108/13555850810890093

von ALTROCK, C. Fuzzy Logic \& Neurofuzzy: Aplications in Business and Finance. New Jersey: Prentice Hall PTR, 1996.

WAGGONER, D. B.; NEELY, A. D.; KENNERLEY, M. $P$. The forces that shape organisational performance measurement systems: an interdisciplinary review. International Journal of Production Economics, v. 60 , n. 1, p. 53-60, 1999. http://dx.doi.org/10.1016/ S0925-5273(98)00201-1

WEBER, C. A. et al. Hybrid analytical hierarchy process model for supplier selection. Industrial Management \& Data Systems, v. 108, n. 1, p. 122-42, 2008. http:// dx.doi.org/10.1108/02635570810844124

WU, W. W.; LEE, Y. T. Developing global managers' competencies using the fuzzy DEMATEL method. Expert Systems with Applications, v. 32, n. 2, p. 499-507, 2007. http://dx.doi.org/10.1016/j.eswa.2005.12.005

YEH, D. Y.; CHENG, C. H.; CHI, M. L. A modified two-tuple FLC model for evatuating the performance of SCM: by the six sigma DMAIC process. Applied Soft Computing, v. 7, p. 1027-1034, 2007. http://dx.doi. org/10.1016/j.asoc.2006.06.008

ZADEH, L. A. Fuzzy sets as basis for a theory of possibility. Fuzzy Sets and Systems, v. 1, n.1, p. 3-28, 1978. http:// dx.doi.org/10.1016/0165-0114(78)90029-5

ZHU, Q.; SARKIS, J.; GENG, Y. Green supply chain management in China: pressures, practices and performance. International Journal of Operations \& Production Management, v. 25, n. 5, p. 449-468, 2005. http://dx.doi.org/10.1108/01443570510593148

ZHU, Q.; SARKIS, J.; LAI, K. H. Confirmation of a measurement model for Green supply chain management practices implementation, International Journal of Production Economics, v. 111, p. 261-273, 2008. http://dx.doi.org/10.1016/j.ijpe.2006.11.029

ZIMMERMANN, H. J. Fuzzy set theory and its applications. 2. ed. Boston: Kluwer Academic, 1991. 